Article

\title{
On the Well-Posedness of A High Order Convective Cahn-Hilliard Type Equations
}

\author{
Giuseppe Maria Coclite ${ }^{1, *}$ and Lorenzo di Ruvo ${ }^{2}$ (D) \\ 1 Dipartimento di Meccanica, Matematica e Management, Politecnico di Bari, 70125 Bari, Italy \\ 2 Dipartimento di Matematica, Università di Bari, 70125 Bari, Italy; lorenzo.diruvo77@gmail.com \\ * Correspondence: giuseppemaria.coclite@poliba.it
}

Received: 3 June 2020; Accepted: 13 July 2020; Published: 16 July 2020

check for updates

\begin{abstract}
High order convective Cahn-Hilliard type equations describe the faceting of a growing surface, or the dynamics of phase transitions in ternary oil-water-surfactant systems. In this paper, we prove the well-posedness of the classical solutions for the Cauchy problem, associated with this equation.
\end{abstract}

Keywords: existence; uniqueness; stability; higher order convective Cahn-Hilliard type equation; Cauchy problem

MSC: 35G25; 35K55

\section{Introduction}

In this paper, we investigate the well-posedness of the following Cauchy problem:

$$
\begin{cases}\partial_{t} u+\kappa \partial_{x} u^{2}-\beta^{2} \partial_{x}^{6} u+\alpha \partial_{x}^{4} u+\delta^{2} \partial_{x}^{4}\left(u^{3}\right)=0, & t>0, \quad x \in \mathbb{R}, \\ u(0, x)=u_{0}(x), & x \in \mathbb{R},\end{cases}
$$

with

$$
\kappa, \beta, \alpha, \delta \in \mathbb{R}, \quad \beta, \delta \neq 0
$$

On the initial datum, we assume

$$
u_{0} \in H^{3}(\mathbb{R}), \quad \int_{\mathbb{R}} u_{0}(x) d x=0
$$

Inspired by [1-12], thanks to (3), we can define the following function:

$$
P_{0}(x)=\int_{-\infty}^{x} u_{0}(y) d y
$$

on which we assume

$$
\left\|P_{0}\right\|_{L^{2}(\mathbb{R})}^{2}=\int_{\mathbb{R}}\left(\int_{-\infty}^{x} u_{0}(y) d y\right)^{2} d x<\infty .
$$

Equation (1) is derived in [13], with $\beta=1, \alpha=-1, \delta=1$, to describe a growing crystalline surface with small slopes that undergoes faceting.

The function $u$ represents the slope of a surface, the constant $\kappa$ is proportional to the deposition strength of an atomic flux, and the overall convective term $\kappa \partial_{x} u^{2}$ stems from the normal impingement of the deposited atoms. The sixth order linear term $\partial_{x}^{6} u$, in (1), comes from a curvature 
dependent regularization, and all other terms represent the anisotropy of the surface energy under surface diffusion.

These equations became popular objects of theoretical studies in the last decade, because they do play an important role in material modeling. Equations based on the Mullins surface diffusion formula, such as in (1), are interesting, as they describe the morphology of crystalline surfaces. As complex interfaces and surfaces are more and more important in highly technological applications such as photovoltaics, the study of analytical aspects also gains momentum (see, [14-22]).

The existence and uniqueness of weak solutions of (1) with periodic boundary conditions is proven in [23], with $\kappa>0$. A similar result is proven in [24], in two space dimensions. In [25], the authors derived stationary solutions of (1), while in [26], the problem for optimal control of (1) is analyzed. In [27], the problem of a global attractor is studied.

Taking $\kappa=0$, (1) reads

$$
\partial_{t} u-\beta^{2} \partial_{x}^{6} u+\alpha \partial_{x}^{4} u+\delta^{2} \partial_{x}^{4}\left(u^{3}\right)=0,
$$

which is a Cahn-Hilliard type equation [28-30]. It describes dynamics of phase transitions in ternary oil-water-surfactant systems. One part of the surfactant is hydrophilic and the other one is lipophilic (and termed amphiphile). In the system, almost pure oil, almost pure water, and microemulsion which consist of a homogeneous, isotropic mixture of oil and water can coexist in equilibrium. The function $u$, in (6), represents the local difference between oil and water concentrations.

In [31] the existence of global attractors is analyzed and in [32] the existence of weak solutions for the initial -boundary-value problem for (6) with degenerate mobility is proven.

Assuming $\beta=\delta=0$ and $\alpha=a^{2} \neq 0$, (1) reads

$$
\partial_{t} u+\kappa \partial_{x} u^{2}+a^{2} \partial_{x}^{4} u=0
$$

(7) arises in interesting physical situations, for example as a model for long waves on a viscous fluid owing down an inclined plane [33] and to derive drift waves in a plasma [34]. Equation (7) was derived also independently by Kuramoto [35-37] as a model for phase turbulence in reaction-diffusion systems and by Sivashinsky [38] as a model for plane flame propagation, describing the combined influence of diffusion and thermal conduction of the gas on the stability of a plane flame front.

Equation (7) also describes incipient instabilities in a variety of physical and chemical systems [39-41]. Moreover, (7), which is also known as the Benney-Lin equation [42,43], was derived by Kuramoto in the study of phase turbulence in the Belousov-Zhabotinsky reaction [44].

The dynamical properties and the existence of exact solutions for (7) were investigated in [45-50]. In [51-53], the control problem for (7) with periodic boundary conditions, and on a bounded interval are studied, respectively. In [54], the problem of global exponential stabilization of (7) with periodic boundary conditions is analyzed. In [55], it is proposed a generalization of optimal control theory for (7), while in [56] the problem of global boundary control of (7) is considered. In [57], the existence of solitonic solutions for (7) is proven. In [58-60], the well-posedness of the Cauchy problem for (7) is proven, using the energy space technique, a priori estimates together with an application of the Cauchy-Kovalevskaya and the fixed point method, respectively. Finally, following [61-63], in [64], the convergence of the solution of (7) to the unique entropy one of the Burgers equation is proven.

The main result of this paper is the following theorem.

Theorem 1. Assume (2), (3), (4) and (5). There exists a unique solution $u$ of (1), such that

$$
\begin{aligned}
& u \in H^{1}((0, T) \times \mathbb{R}) \cap L^{\infty}\left(0, T ; H^{3}(\mathbb{R})\right), \\
& \int_{\mathbb{R}} u(t, x) d x=0, \quad t>0 .
\end{aligned}
$$


Moreover, if $u_{1}$ and $u_{2}$ are two solutions of (1), we have that

$$
\left\|u_{1}(t, \cdot)-u_{2}(t, \cdot)\right\|_{L^{2}(\mathbb{R})} \leq e^{C(T) t}\left\|u_{1,0}-u_{2,0}\right\|_{L^{2}(\mathbb{R})},
$$

for some suitable $C(T)>0$, and every $0 \leq t \leq T$.

Compared to $[23,31,65]$, we do not have additional assumption on the constants. Theorem 1 gives the global well-posedness of the classical solution of the Cauchy problem of (1). The argument of Theorem 1 relies on deriving suitable a priori estimates together with an application of the Fixed Point Theorem. Indeed, to make the argument completely rigorous we should approximate the initial datum with an analytic one. Considering the semigroup $S_{t}$ generated by the operator $-\beta^{2} \partial_{x}^{6} u$ we rewrite (1) using the Duhamel formula

$$
u(t, \cdot)=S_{t} u_{0}-\int_{0}^{t} S_{t-s}\left(\kappa \partial_{x} u^{2} u+\alpha \partial_{x}^{4} u+\delta^{2} \partial_{x}^{4}\left(u^{3}\right)\right)(s, \cdot) d s .
$$

Then standard arguments and the Fixed Point Theorem would give us the local in time existence of analytic solutions. The estimates will guarantee that these approximate solutions are indeed global in time and allow us to come back to the original initial datum.

The paper is organized as follows. In Section 2, we prove some a priori estimates of (1). Those play a key role in the proof of our main result, which is given in Section 3.

\section{A Priori Estimates}

In this section, we prove some a priori estimates on $u$. We denote with $C_{0}$ the constants which depend only on the initial data, and with $C(T)$, the constants which depend also on $T$.

We begin by proving the following lemma.

Lemma 1. For each $t>0$, we have that (9).

Proof. Integrating (1) on $\mathbb{R}$, we have that

$$
\int_{\mathbb{R}} \partial_{t} u(t, x) d x=\frac{d}{d t} \int_{\mathbb{R}} u(t, x) d x=0
$$

(9) follows from (3) and (11).

Remark 1. In light of (9), we can consider the following function:

$$
P(t, x)=\int_{-\infty}^{x} u(t, y) d y .
$$

Moreover, again by (9), we have that

$$
P(t,-\infty)=P(t, \infty)=0 .
$$

Lemma 2. Let $T>0$. There exists a constant $C(T)>0$, such that

$$
\begin{aligned}
\|P(t, \cdot)\|_{L^{2}(\mathbb{R})}^{2} & +2 \beta^{2} e^{t} \int_{0}^{t} e^{-s}\left\|\partial_{x}^{2} u(s, \cdot)\right\|_{L^{2}(\mathbb{R})}^{2} d s \\
& +2 \delta^{2} e^{t} \int_{0}^{t} e^{-s}\left\|u(s, \cdot) \partial_{x} u(s, \cdot)\right\|_{L^{2}(\mathbb{R})}^{2} d s \\
\leq & C(T)+C(T) \int_{0}^{t}\|u(s, \cdot)\|_{L^{4}(\mathbb{R})}^{4} d s+C(T) \int_{0}^{t}\left\|\partial_{x} u(s, \cdot)\right\|_{L^{2}(\mathbb{R})}^{2} d s,
\end{aligned}
$$


for every $0 \leq t \leq T$.

Proof. Let $0 \leq t \leq T$. We begin by observing that

$$
\partial_{x} u^{3}=3 u^{2} \partial_{x} u \text {. }
$$

Integrating (1) on $(-\infty, x)$, we have that

$$
\int_{-\infty}^{x} \partial_{t} u d y+\kappa u^{2}-\beta^{2} \partial_{x}^{5} u+\alpha \partial_{x}^{3} u+\delta^{2} \partial_{x}^{3}\left(u^{3}\right)=0 .
$$

Differentiating (12) with respect to $t$, we obtain that

$$
\partial_{t} P(t, x)=\frac{d}{d t} \int_{-\infty}^{x} u(t, y) d y=\int_{-\infty}^{x} \partial_{t} u(t, y) d y .
$$

From (16) and (17)

$$
\partial_{t} P+\kappa u^{2}-\beta^{2} \partial_{x}^{5} u+\alpha \partial_{x}^{3} u+\delta^{2} \partial_{x}^{3}\left(u^{3}\right)=0 .
$$

Observe that by (13) and (18),

$$
\begin{aligned}
-2 \beta^{2} \int_{\mathbb{R}} P \partial_{x}^{5} u d x & =2 \beta^{2} \int_{\mathbb{R}} \partial_{x} P \partial_{x}^{4} u d x=\beta^{2} \int_{\mathbb{R}} u \partial_{x}^{4} u d x \\
& =-2 \beta^{2} \int_{\mathbb{R}} \partial_{x} u \partial_{x}^{3} u d x=2 \beta^{2}\left\|\partial_{x}^{2} u(t, \cdot)\right\|_{L^{2}(\mathbb{R})}^{2} \\
2 \alpha \int_{\mathbb{R}} P \partial_{x}^{3} u d x & =-2 \alpha \int_{\mathbb{R}} \partial_{x} P \partial_{x}^{2} u d x=-2 \alpha \int_{\mathbb{R}} u \partial_{x}^{2} u d x=2 \alpha\left\|\partial_{x} u(t, \cdot)\right\|_{L^{2}(\mathbb{R})}^{2} .
\end{aligned}
$$

Moreover, by (15) and (16),

$$
\begin{aligned}
2 \delta^{2} \int_{\mathbb{R}} P \partial_{x}^{3}\left(u^{3}\right) d x & =-2 \delta^{2} \int_{\mathbb{R}} \partial_{x} P \partial_{x}^{2}\left(u^{3}\right) d x=-2 \delta^{2} \int_{\mathbb{R}} u \partial_{x}^{2}\left(u^{3}\right) d x \\
& =2 \delta^{2} \int_{\mathbb{R}} \partial_{x} u \partial_{x}\left(u^{3}\right) d x=6 \delta^{2}\left\|u(t, \cdot) \partial_{x} u(t, \cdot)\right\|_{L^{2}(\mathbb{R})}^{2}
\end{aligned}
$$

Therefore, multiplying (18) by $2 P$, an integration on $\mathbb{R},(19)$ and (20) give

$$
\begin{gathered}
\frac{d}{d t}\|P(t, \cdot)\|_{L^{2}(\mathbb{R})}^{2}+2 \beta^{2}\left\|\partial_{x}^{2} u(t, \cdot)\right\|_{L^{2}(\mathbb{R})}^{2}+6 \delta^{2}\left\|u(t, \cdot) \partial_{x} u(t, \cdot)\right\|_{L^{2}(\mathbb{R})}^{2} \\
=-2 \kappa \int_{\mathbb{R}} P u^{2} d x-2 \alpha\left\|\partial_{x} u(t, \cdot)\right\|_{L^{2}(\mathbb{R})}^{2} .
\end{gathered}
$$

Due to the Young inequality,

$$
2|\kappa| \int_{\mathbb{R}}|P| u^{2} d x \leq\|P(t, \cdot)\|_{L^{2}(\mathbb{R})}^{2}+\kappa^{2}\|u(t, \cdot)\|_{L^{4}(\mathbb{R})}^{4} .
$$

It follows from (21) that

$$
\begin{gathered}
\frac{d}{d t}\|P(t, \cdot)\|_{L^{2}(\mathbb{R})}^{2}+2 \beta^{2}\left\|\partial_{x}^{2} u(t, \cdot)\right\|_{L^{2}(\mathbb{R})}^{2}+6 \delta^{2}\left\|u(t, \cdot) \partial_{x} u(t, \cdot)\right\|_{L^{2}(\mathbb{R})}^{2} \\
\leq\|P(t, \cdot)\|_{L^{2}(\mathbb{R})}^{2}+\kappa^{2}\|u(t, \cdot)\|_{L^{4}(\mathbb{R})}^{4}+2|\alpha|\left\|\partial_{x} u(t, \cdot)\right\|_{L^{2}(\mathbb{R})}^{2} \\
\leq\|P(t, \cdot)\|_{L^{2}(\mathbb{R})}^{2}+C_{0}\|u(t, \cdot)\|_{L^{4}(\mathbb{R})}^{4}+C_{0}\left\|\partial_{x} u(t, \cdot)\right\|_{L^{2}(\mathbb{R})}^{2} .
\end{gathered}
$$


Therefore, by the Gronwall Lemma and (5), we have that

$$
\begin{aligned}
& \|P(t, \cdot)\|_{L^{2}(\mathbb{R})}^{2}+2 \beta^{2} e^{t} \int_{0}^{t} e^{-s}\left\|\partial_{x}^{2} u(s, \cdot)\right\|_{L^{2}(\mathbb{R})}^{2} d s+6 \delta^{2} e^{t} \int_{0}^{t} e^{-s}\left\|u(s, \cdot) \partial_{x} u(s, \cdot)\right\|_{L^{2}(\mathbb{R})}^{2} d s \\
& \quad \leq C_{0}+C_{0} e^{t} \int_{0}^{t} e^{-s}\|u(s, \cdot)\|_{L^{4}(\mathbb{R})}^{4} d s+C_{0} e^{t} \int_{0}^{t} e^{-s}\left\|\partial_{x} u(s, \cdot)\right\|_{L^{2}(\mathbb{R})}^{2} d s \\
& \quad \leq C(T)+C(T) \int_{0}^{t}\|u(s, \cdot)\|_{L^{4}(\mathbb{R})}^{4} d s+C(T) \int_{0}^{t}\left\|\partial_{x} u(s, \cdot)\right\|_{L^{2}(\mathbb{R})}^{2} d s,
\end{aligned}
$$

which gives (14).

Lemma 3. Let $T>0$. There exists a constant $C(T)>0$, such that

$$
\begin{aligned}
\frac{\beta^{2}}{6}\left\|\partial_{x} u(t, \cdot)\right\|_{L^{2}(\mathbb{R})}^{2}+\frac{\delta^{2}}{4}\|u(t, \cdot)\|_{L^{4}(\mathbb{R})}^{2} & \leq C(T), \\
\|P(t, \cdot)\|_{L^{2}(\mathbb{R})} & \leq C(T), \\
\|u(t, \cdot)\|_{L^{2}(\mathbb{R})} & \leq C(T), \\
\int_{0}^{t}\left\|\partial_{x}^{2} u(s, \cdot)\right\|_{L^{2}(\mathbb{R})}^{2} d s & \leq C(T), \\
\int_{0}^{t}\left\|u(s, \cdot) \partial_{x} u(s, \cdot)\right\|_{L^{2}(\mathbb{R})}^{2} d s & \leq C(T), \\
\int_{0}^{t} \int_{\mathbb{R}}\left[\beta^{2}\left(\partial_{x}^{4} u\right)^{2}-\delta^{2} \partial_{x}^{3}\left(u^{3}\right)\right]^{2} d s d x & \leq C(T), \\
\|P\|_{L^{\infty}((0, T) \times \mathbb{R})} & \leq C(T) \\
\|u\|_{L^{\infty}((0, T) \times \mathbb{R})} & \leq C(T), \\
\int_{0}^{t}\left\|\partial_{x} u(s, \cdot)\right\|_{L^{4}(\mathbb{R})}^{4} d s & \leq C(T),
\end{aligned}
$$

for every $0 \leq t \leq T$.

Proof. Let $0 \leq t \leq T$. Consider an real constant $A$, which will specified later. Multiplying (1) by

$$
-\beta^{2} \partial_{x}^{2} u+\delta^{2} u^{3}+A u
$$

we have that

$$
\begin{aligned}
\left(-\beta^{2} \partial_{x}^{2} u+\delta^{2} u^{3}+A u\right) \partial_{t} u & +2 \kappa\left(-\beta^{2} \partial_{x}^{2} u+\delta^{2} u^{3}+A u\right) u \partial_{x} u \\
& -\beta^{2}\left(-\beta^{2} \partial_{x}^{2} u+\delta^{2} u^{3}+A u\right) \partial_{x}^{6} u \\
& +\alpha\left(-\beta^{2} \partial_{x}^{2} u+\delta^{2} u^{3}+A u\right) \partial_{x}^{4} u \\
& +\delta^{2}\left(-\beta^{2} \partial_{x}^{2} u+\delta^{2} u^{3}+A u\right) \partial_{x}^{4}\left(u^{3}\right)=0
\end{aligned}
$$

Observe that

$$
\begin{aligned}
& \int_{\mathbb{R}}\left(-\beta^{2} \partial_{x}^{2} u+\delta^{2} u^{3}+A u\right) \partial_{t} u d x \\
& \quad=\frac{d}{d t}\left(\frac{\beta^{2}}{2}\left\|\partial_{x} u(t, \cdot)\right\|_{L^{2}(\mathbb{R})}^{2}+\frac{\delta^{2}}{4}\|u(t, \cdot)\|_{L^{4}(\mathbb{R})}^{4}+\frac{A}{2}\|u(t, \cdot)\|_{L^{2}(\mathbb{R})}^{2}\right), \\
& 2 \kappa \int_{\mathbb{R}}\left(-\beta^{2} \partial_{x}^{2} u+\delta^{2} u^{3}+A u\right) u \partial_{x} u d x=-2 \beta^{2} \kappa \int_{\mathbb{R}} u \partial_{x} u \partial_{x}^{2} u d x, \\
& -\beta^{2} \int_{\mathbb{R}}\left(-\beta^{2} \partial_{x}^{2} u+\delta^{2} u^{3}+A u\right) \partial_{x}^{6} u d x
\end{aligned}
$$




$$
\begin{gathered}
=-\beta^{4} \int_{\mathbb{R}} \partial_{x}^{3} u \partial_{x}^{5} u d x+\beta^{2} \delta^{2} \int_{\mathbb{R}} \partial_{x}\left(u^{3}\right) \partial_{x}^{5} u d x+A \beta^{2} \int_{\mathbb{R}} \partial_{x} u \partial_{x}^{5} u d x \\
=\beta^{4} \int_{\mathbb{R}}\left(\partial_{x}^{4} u\right)^{2} d x-\beta^{2} \delta^{2} \int_{\mathbb{R}} \partial_{x}^{2}\left(u^{3}\right) \partial_{x}^{4} u d x-A \beta^{2} \int_{\mathbb{R}} \partial_{x}^{2} u \partial_{x}^{4} u d x \\
=\beta^{4} \int_{\mathbb{R}}\left(\partial_{x}^{4} u\right)^{2} d x-\beta^{2} \delta^{2} \int_{\mathbb{R}} \partial_{x}^{2}\left(u^{3}\right) \partial_{x}^{4} u d x+A \beta^{2}\left\|\partial_{x}^{3} u(t, \cdot)\right\|_{L^{2}(\mathbb{R})^{\prime}}^{2} \\
\alpha \int_{\mathbb{R}}\left(-\beta^{2} \partial_{x}^{2} u+\delta^{2} u^{3}+A u\right) \partial_{x}^{4} u d x \\
=\alpha \beta^{2}\left\|\partial_{x}^{3} u(t, \cdot)\right\|_{L^{2}(\mathbb{R})}^{2}-\alpha \delta^{2} \int_{\mathbb{R}} \partial_{x}^{3} u \partial_{x}\left(u^{3}\right) d x-A \alpha \int_{\mathbb{R}} \partial_{x} u \partial_{x}^{3} u d x \\
=\alpha \beta^{2}\left\|\partial_{x}^{3} u(t, \cdot)\right\|_{L^{2}(\mathbb{R})}^{2}+\alpha \delta^{2} \int_{\mathbb{R}} \partial_{x}^{2} u \partial_{x}^{2}\left(u^{3}\right) d x+A \alpha \partial_{x}^{2} u(t, \cdot) \|_{L^{2}(\mathbb{R})^{\prime}}^{2} \\
\delta^{2} \int_{\mathbb{R}}\left(-\beta^{2} \partial_{x}^{2} u+\delta^{2} u^{3}+A u\right) \partial_{x}^{4}\left(u^{3}\right) d x \\
=\delta^{2} \beta^{2} \int_{\mathbb{R}} \partial_{x}^{3} u \partial_{x}^{3}\left(u^{3}\right) d x-\delta^{4} \int_{\mathbb{R}} \partial_{x} u^{3} \partial_{x}^{3}\left(u^{3}\right) d x-A \delta^{2} \int_{\mathbb{R}} \partial_{x} u \partial_{x}^{3}\left(u^{3}\right) d x \\
=-\delta \beta^{2} \int_{\mathbb{R}} \partial_{x}^{4} u \partial_{x}^{2}\left(u^{3}\right) d x+\delta^{4} \int_{\mathbb{R}}\left[\partial_{x}^{2}\left(u^{3}\right)\right]^{2} d x+A \delta^{2} \int_{\mathbb{R}} \partial_{x}^{2} u \partial_{x}^{2}\left(u^{3}\right) d x .
\end{gathered}
$$

Consequently, integrating (31) on $\mathbb{R}$, by (32), we have that

$$
\begin{aligned}
\frac{d}{d t}\left(\frac{\beta^{2}}{2}\right. & \left.\left\|\partial_{x} u(t, \cdot)\right\|_{L^{2}(\mathbb{R})}^{2}+\frac{\delta^{2}}{4}\|u(t, \cdot)\|_{L^{4}(\mathbb{R})}^{4}+\frac{A}{2}\|u(t, \cdot)\|_{L^{2}(\mathbb{R})}^{2}\right) \\
& +\beta^{4} \int_{\mathbb{R}}\left(\partial_{x}^{4} u\right)^{2} d x-2 \beta^{2} \delta^{2} \int_{\mathbb{R}} \partial_{x}^{4} u \partial_{x}^{2}\left(u^{3}\right) d x+\delta^{4} \int_{\mathbb{R}}\left[\partial_{x}^{2}\left(u^{3}\right)\right]^{2} d x \\
= & -\beta^{2}(A+\alpha)\left\|\partial_{x}^{3} u(t, \cdot)\right\|_{L^{2}(\mathbb{R})}^{2}-\delta^{2}(A+\alpha) \int_{\mathbb{R}} \partial_{x}^{2} u \partial_{x}^{2}\left(u^{3}\right) d x \\
& -A \alpha\left\|\partial_{x}^{2} u(t, \cdot)\right\|_{L^{2}(\mathbb{R})}^{2}+2 \beta^{2} \kappa \int_{\mathbb{R}} u \partial_{x} u \partial_{x}^{2} u d x .
\end{aligned}
$$

Taking

$$
A=-\alpha,
$$

by (33), we have that

$$
\begin{aligned}
& \frac{d}{d t}\left(\frac{\beta^{2}}{2}\left\|\partial_{x} u(t, \cdot)\right\|_{L^{2}(\mathbb{R})}^{2}+\frac{\delta^{2}}{4}\|u(t, \cdot)\|_{L^{4}(\mathbb{R})}^{4}-\frac{\alpha}{2}\|u(t, \cdot)\|_{L^{2}(\mathbb{R})}^{2}\right) \\
& \quad+\beta^{4} \int_{\mathbb{R}}\left(\partial_{x}^{4} u\right)^{2} d x-2 \beta^{2} \delta^{2} \int_{\mathbb{R}} \partial_{x}^{4} u \partial_{x}^{2}\left(u^{3}\right) d x+\delta^{4} \int_{\mathbb{R}}\left[\partial_{x}^{2}\left(u^{3}\right)\right]^{2} d x \\
& =\alpha^{2}\left\|\partial_{x}^{2} u(t, \cdot)\right\|_{L^{2}(\mathbb{R})}^{2}+2 \beta^{2} \kappa \int_{\mathbb{R}} u \partial_{x} u \partial_{x}^{2} u d x .
\end{aligned}
$$

Observe that

$$
\begin{gathered}
\beta^{4} \int_{\mathbb{R}}\left(\partial_{x}^{4} u\right)^{2} d x-2 \beta^{2} \delta^{2} \int_{\mathbb{R}} \partial_{x}^{4} u \partial_{x}^{2}\left(u^{3}\right) d x+\delta^{4} \int_{\mathbb{R}}\left[\partial_{x}^{2}\left(u^{3}\right)\right]^{2} d x \\
=\int_{\mathbb{R}}\left[\beta^{2}\left(\partial_{x}^{4} u\right)^{2}-\delta^{2} \partial_{x}^{2}\left(u^{3}\right)\right]^{2} d x .
\end{gathered}
$$

Consequently, by (35),

$$
\begin{gathered}
\frac{d}{d t}\left(\frac{\beta^{2}}{2}\left\|\partial_{x} u(t, \cdot)\right\|_{L^{2}(\mathbb{R})}^{2}+\frac{\delta^{2}}{4}\|u(t, \cdot)\|_{L^{4}(\mathbb{R})}^{4}-\frac{\alpha}{2}\|u(t, \cdot)\|_{L^{2}(\mathbb{R})}^{2}\right) \\
+\int_{\mathbb{R}}\left[\beta^{2}\left(\partial_{x}^{4} u\right)^{2}-\delta^{2} \partial_{x}^{3}\left(u^{3}\right)\right]^{2} d x
\end{gathered}
$$




$$
=\alpha^{2}\left\|\partial_{x}^{2} u(t, \cdot)\right\|_{L^{2}(\mathbb{R})}^{2}+2 \beta^{2} \kappa \int_{\mathbb{R}} u \partial_{x} u \partial_{x}^{2} u d x
$$

Due to the Young inequality,

$$
2 \beta^{2}|\kappa| \int_{\mathbb{R}}\left|u \partial_{x} u\left\|\partial_{x}^{2} u \mid d x \leq \kappa^{2}\right\| u(t, \cdot) \partial_{x} u(t, \cdot)\left\|_{L^{2}(\mathbb{R})}^{2}+\beta^{4}\right\| \partial_{x}^{2} u(t, \cdot) \|_{L^{2}(\mathbb{R})}^{2} .\right.
$$

Therefore, by (36),

$$
\begin{aligned}
\frac{d}{d t}( & \left.\frac{\beta^{2}}{2}\left\|\partial_{x} u(t, \cdot)\right\|_{L^{2}(\mathbb{R})}^{2}+\frac{\delta^{2}}{4}\|u(t, \cdot)\|_{L^{4}(\mathbb{R})}^{4}-\frac{\alpha}{2}\|u(t, \cdot)\|_{L^{2}(\mathbb{R})}^{2}\right) \\
& +\int_{\mathbb{R}}\left[\beta^{2}\left(\partial_{x}^{4} u\right)^{2}-\delta^{2} \partial_{x}^{3}\left(u^{3}\right)\right]^{2} d x \\
\leq & \left(\alpha^{2}+\beta^{4}\right)\left\|\partial_{x}^{2} u(t, \cdot)\right\|_{L^{2}(\mathbb{R})}^{2}+\kappa^{2}\left\|u(t, \cdot) \partial_{x} u(t, \cdot)\right\|_{L^{2}(\mathbb{R})}^{2} \\
\leq & C_{0} \beta^{2}\left\|\partial_{x}^{2} u(t, \cdot)\right\|_{L^{2}(\mathbb{R})}^{2}+C_{0} \delta^{4}\left\|u(t, \cdot) \partial_{x} u(t, \cdot)\right\|_{L^{2}(\mathbb{R})}^{2} .
\end{aligned}
$$

Integrating on $(0, t)$, by (3), we have that

$$
\begin{aligned}
\frac{\beta^{2}}{2}\left\|\partial_{x} u(t, \cdot)\right\|_{L^{2}(\mathbb{R})}^{2}+\frac{\delta^{2}}{4}\|u(t, \cdot)\|_{L^{4}(\mathbb{R})}^{4}-\frac{\alpha}{2}\|u(t, \cdot)\|_{L^{2}(\mathbb{R})}^{2} \\
\quad+\int_{0}^{t} \int_{\mathbb{R}}\left[\beta^{2}\left(\partial_{x}^{4} u\right)^{2}-\delta^{2} \partial_{x}^{3}\left(u^{3}\right)\right]^{2} d s d x \\
\leq C_{0}+C_{0} \beta^{2} \int_{0}^{t}\left\|\partial_{x}^{2} u(s, \cdot)\right\|_{L^{2}(\mathbb{R})}^{2}+C_{0} \delta^{4} \int_{0}^{t}\left\|u(s, \cdot) \partial_{x} u(s, \cdot)\right\|_{L^{2}(\mathbb{R})}^{2} d s .
\end{aligned}
$$

Therefore, we obtain that

$$
\begin{aligned}
\frac{\beta^{2}}{2}\left\|\partial_{x} u(t, \cdot)\right\|_{L^{2}(\mathbb{R})}^{2}+\frac{\delta^{2}}{4}\|u(t, \cdot)\|_{L^{4}(\mathbb{R})}^{4} & \\
& +\int_{0}^{t} \int_{\mathbb{R}}\left[\beta^{2}\left(\partial_{x}^{4} u\right)^{2}-\delta^{2} \partial_{x}^{3}\left(u^{3}\right)\right]^{2} d s d x \\
\leq & C_{0}+C_{0} \beta^{2} \int_{0}^{t}\left\|\partial_{x}^{2} u(s, \cdot)\right\|_{L^{2}(\mathbb{R})}^{2}+C_{0} \delta^{4} \int_{0}^{t}\left\|u(s, \cdot) \partial_{x} u(s, \cdot)\right\|_{L^{2}(\mathbb{R})}^{2} d s+\frac{\alpha}{2}\|u(t, \cdot)\|_{L^{2}(\mathbb{R})}^{2} \\
\leq & C_{0}+C_{0} \beta^{2} \int_{0}^{t}\left\|\partial_{x}^{2} u(s, \cdot)\right\|_{L^{2}(\mathbb{R})}^{2}+C_{0} \delta^{4} \int_{0}^{t}\left\|u(s, \cdot) \partial_{x} u(s, \cdot)\right\|_{L^{2}(\mathbb{R})}^{2} d s+C_{0}\|u(t, \cdot)\|_{L^{2}(\mathbb{R})}^{2} .
\end{aligned}
$$

Observe that by (12) and (13),

$$
C_{0}\|u(t, \cdot)\|_{L^{2}(\mathbb{R})}^{2}=C_{0} \int_{\mathbb{R}} u u d x=-C_{0} \int_{\mathbb{R}} P \partial_{x} u d x .
$$

Therefore, by the Young inequality,

$$
\begin{aligned}
C_{0}\|u(t, \cdot)\|_{L^{2}(\mathbb{R})}^{2} & \leq 2 \int_{\mathbb{R}}\left|\frac{\sqrt{3} C_{0} P}{2 \beta}\right|\left|\frac{\beta \partial_{x} u}{\sqrt{3}}\right| d x \\
& \leq C_{0}\|P(t, \cdot)\|_{L^{2}(\mathbb{R})}^{2}+\frac{\beta^{2}}{3}\left\|\partial_{x} u(t, \cdot)\right\|_{L^{2}(\mathbb{R})}^{2} .
\end{aligned}
$$

If follows from (14) and (37) that

$$
\frac{\beta^{2}}{6}\left\|\partial_{x} u(t, \cdot)\right\|_{L^{2}(\mathbb{R})}^{2}+\frac{\delta^{2}}{4}\|u(t, \cdot)\|_{L^{4}(\mathbb{R})}^{4}
$$




$$
\begin{aligned}
& +\int_{0}^{t} \int_{\mathbb{R}}\left[\beta^{2}\left(\partial_{x}^{4} u\right)^{2}-\delta^{2} \partial_{x}^{3}\left(u^{3}\right)\right]^{2} d s d x \\
\leq & C_{0}+C_{0}\|P(t, \cdot)\|_{L^{2}(\mathbb{R})}^{2}+C_{0} \beta^{2} \int_{0}^{t}\left\|\partial_{x}^{2} u(s, \cdot)\right\|_{L^{2}(\mathbb{R})}^{2} d s \\
& +C_{0} \delta^{4} \int_{0}^{t}\left\|u(s, \cdot) \partial_{x} u(s, \cdot)\right\|_{L^{2}(\mathbb{R})}^{2} d s \\
\leq & C_{0}+C_{0}\|P(t, \cdot)\|_{L^{2}(\mathbb{R})}^{2}+C_{0} \beta^{2} e^{t} \int_{0}^{t} e^{-s}\left\|\partial_{x}^{2} u(s, \cdot)\right\|_{L^{2}(\mathbb{R})}^{2} d s \\
& +C_{0} \delta^{4} e^{t} \int_{0}^{t} e^{s}\left\|u(s, \cdot) \partial_{x} u(s, \cdot)\right\|_{L^{2}(\mathbb{R})}^{2} d s \\
\leq & C(T)+C(T) \int_{0}^{t}\left\|\partial_{x} u(s, \cdot)\right\|_{L^{2}(\mathbb{R})}^{2} d s+C(T) \int_{0}^{t}\|u(s, \cdot)\|_{L^{4}(\mathbb{R})}^{4} d s \\
\leq & C(T)+C(T)\left(\frac{\beta^{2}}{6} \int_{0}^{t}\left\|\partial_{x} u(s, \cdot)\right\|_{L^{2}(\mathbb{R})}^{2} d s+\frac{\delta^{2}}{4} \int_{0}^{t}\|u(s, \cdot)\|_{L^{4}(\mathbb{R})}^{4} d s\right) .
\end{aligned}
$$

Therefore, we have that

$$
\begin{aligned}
& \frac{\beta^{2}}{6}\left\|\partial_{x} u(t, \cdot)\right\|_{L^{2}(\mathbb{R})}^{2}+\frac{\delta^{2}}{4}\|u(t, \cdot)\|_{L^{4}(\mathbb{R})}^{4} \\
& \quad \leq C(T)+C(T)\left(\frac{\beta^{2}}{6} \int_{0}^{t}\left\|\partial_{x} u(s, \cdot)\right\|_{L^{2}(\mathbb{R})}^{2} d s+\frac{\delta^{2}}{4} \int_{0}^{t}\|u(s, \cdot)\|_{L^{4}(\mathbb{R})}^{4} d s\right) .
\end{aligned}
$$

The Gronwall Lemma and (5) give (22).

(23)-(27) follow from (14), (22) and (38).

We prove (28). Thanks to (12), (13), (23)-(27) and the Hölder inequality,

$$
\begin{aligned}
P^{2}(t, x)=2 \int_{-\infty}^{x} P \partial_{x} P d y & =2 \int_{-\infty}^{x} P u d x \leq 2 \int_{\mathbb{R}}|P \| u| d x \\
& \leq 2\|P(t, \cdot)\|_{L^{2}(\mathbb{R})}\|u(t, \cdot)\|_{L^{2}(\mathbb{R})} \leq C(T)
\end{aligned}
$$

Therefore,

$$
\|P\|_{L^{\infty}((0, T) \times \mathbb{R})}^{2} \leq C(T),
$$

which gives (28).

We prove (29). Thanks to (22) and the Hölder,

$$
\begin{aligned}
|u(t, x)|^{3} & =3\left|\int_{-\infty}^{x} u^{2} \partial_{x} u d y\right| \leq 3 \int_{\mathbb{R}} u^{2}\left|\partial_{x} u\right| d x \\
& \leq 3\|u(t, \cdot)\|_{L^{4}(\mathbb{R})}^{2}\left\|\partial_{x} u(t, \cdot)\right\|_{L^{2}(\mathbb{R})} \leq C(T) .
\end{aligned}
$$

Therefore,

$$
\|u\|_{L^{\infty}((0, T) \times \mathbb{R})}^{3} \leq C(T) .
$$

which gives (29).

Finally, we prove (30). We begin by observing that ([66], Lemma 2.3) says that

$$
\left\|\partial_{x} u(t, \cdot)\right\|_{L^{4}(\mathbb{R})}^{4} \leq 6\left(\|u(t, \cdot)\|_{L^{2}(\mathbb{R})}^{2}+\left\|\partial_{x} u(t, \cdot)\right\|_{L^{2}(\mathbb{R})}^{2}\right)\left\|\partial_{x}^{2} u(t, \cdot)\right\|_{L^{2}(\mathbb{R})}^{2} .
$$

Therefore, by (22) and (23),

$$
\left\|\partial_{x} u(t, \cdot)\right\|_{L^{4}(\mathbb{R})}^{4} \leq C(T)\left\|\partial_{x}^{2} u(t, \cdot)\right\|_{L^{2}(\mathbb{R})}^{2} .
$$

Integrating (39) on $(0, t)$, by (23), we have (30). 
Lemma 4. Let $T>0$. There exists a constant $C(T)>0$, such that

$$
\begin{aligned}
& \int_{0}^{t}\left\|\partial_{x}^{4} u(s, \cdot)\right\|_{L^{2}(\mathbb{R})}^{2} d s \leq C(T), \\
& \int_{0}^{t}\left\|\partial_{x}^{3} u(s, \cdot)\right\|_{L^{2}(\mathbb{R})}^{2} d s \leq C(T),
\end{aligned}
$$

for every $0 \leq t \leq T$.

Proof. Let $0 \leq t \leq T$. We begin by observing that

$$
\partial_{x}^{2}\left(u^{3}\right)=6 u\left(\partial_{x} u\right)^{2}+3 u^{2} \partial_{x}^{2} u .
$$

By (27), we have that

$$
\begin{aligned}
\beta^{4} \int_{0}^{t}\left\|\partial_{x}^{4} u(s, \cdot)\right\|_{L^{2}(\mathbb{R})}^{2} d s \leq & C(T)-\delta^{4} \int_{0}^{t} \int_{\mathbb{R}}\left[\partial_{x}^{2}\left(u^{3}\right)\right]^{2} d s d x \\
& +2 \beta^{2} \delta^{2} \int_{0}^{t} \int_{\mathbb{R}} \partial_{x}^{4} u \partial_{x}^{2}\left(u^{3}\right) d s d x
\end{aligned}
$$

Due to the Young inequality,

$$
2 \beta^{2} \delta^{2} \int_{0}^{t} \int_{\mathbb{R}}\left|\partial_{x}^{4} u\left\|\partial_{x}^{2}\left(u^{3}\right) \mid d s d x \leq \frac{\beta^{4}}{2}\right\| \partial_{x}^{4} u(t, \cdot) \|_{L^{2}(\mathbb{R})}^{2}+2 \delta^{4} \int_{0}^{t} \int_{\mathbb{R}}\left[\partial_{x}^{2}\left(u^{3}\right)\right]^{2} d s d x .\right.
$$

Therefore, by (43),

$$
\frac{\beta^{4}}{2}\left\|\partial_{x}^{4} u(t, \cdot)\right\|_{L^{2}(\mathbb{R})}^{2} \leq C(T)+\delta^{4} \int_{0}^{t} \int_{\mathbb{R}}\left[\partial_{x}^{2}\left(u^{3}\right)\right]^{2} d s d x .
$$

Observe that (42),

$$
\begin{aligned}
\int_{\mathbb{R}}\left[\partial_{x}^{2}\left(u^{3}\right)\right]^{2} d x & =36 \int_{\mathbb{R}} u^{2}\left(\partial_{x} u\right)^{4} d x+9\left\|u^{2}(t, \cdot) \partial_{x}^{2} u(t, \cdot)\right\|_{L^{2}(\mathbb{R})}^{2}+36 \int_{\mathbb{R}} u^{3}\left(\partial_{x} u\right)^{2} \partial_{x}^{2} u d x \\
& =36 \int_{\mathbb{R}} u^{2}\left(\partial_{x} u\right)^{4} d x+9\left\|u^{2}(t, \cdot) \partial_{x}^{2} u(t, \cdot)\right\|_{L^{2}(\mathbb{R})}^{2}-36 \int_{\mathbb{R}} u^{2}\left(\partial_{x} u\right)^{4} d x \\
& =9\left\|u^{2}(t, \cdot) \partial_{x}^{2} u(t, \cdot)\right\|_{L^{2}(\mathbb{R})}^{2}=9 \int_{\mathbb{R}} u^{4}\left(\partial_{x}^{2} u\right)^{2} d x .
\end{aligned}
$$

Therefore, by (23) and (29),

$$
\begin{aligned}
\int_{\mathbb{R}}\left[\partial_{x}^{2}\left(u^{3}\right)\right]^{2} d x & \leq 9\|u\|_{L^{\infty}((0, T) \times \mathbb{R})}^{4}\left\|\partial_{x}^{2} u(t, \cdot)\right\|_{L^{2}(\mathbb{R})}^{2} \\
& \leq C(T)\left\|\partial_{x}^{2} u(t, \cdot)\right\|_{L^{2}(\mathbb{R})}^{2} .
\end{aligned}
$$

Integrating $(45)$ on $(0, t)$, by $(23)$, we have that

$$
\int_{0}^{t} \int_{\mathbb{R}}\left[\partial_{x}^{2}\left(u^{3}\right)\right]^{2} d s d x \leq C(T) \int_{0}^{t}\left\|\partial_{x}^{2} u(s, \cdot)\right\|^{2} d s \leq C(T) .
$$

(40) follows from (44) and (46).

Finally, we prove (41). We begin by observing that

$$
\left\|\partial_{x}^{3} u(t, \cdot)\right\|_{L^{2}(\mathbb{R})}^{2}=\int_{\mathbb{R}} \partial_{x}^{3} u \partial_{x}^{3} u d x=-\int_{\mathbb{R}} \partial_{x}^{2} u \partial_{x}^{4} u d x .
$$


Therefore, by the Young inequality,

$$
\left\|\partial_{x}^{3} u(t, \cdot)\right\|_{L^{2}(\mathbb{R})}^{2} \leq \int_{\mathbb{R}}\left|\partial_{x}^{2} u\left\|\partial_{x}^{4} u \mid d x \leq \frac{1}{2}\right\| \partial_{x}^{2} u(t, \cdot)\left\|_{L^{2}(\mathbb{R})}^{2}+\frac{1}{2}\right\| \partial_{x}^{4} u(t, \cdot) \|_{L^{2}(\mathbb{R})}^{2} .\right.
$$

(23), (40) and an integration on $(0, t)$ of (47) give (41).

Lemma 5. Fix $T>0$. There exists a constant $C(T)>0$, such that

$$
\begin{array}{r}
\left\|\partial_{x} u\right\|_{L^{\infty}((0, T) \times \mathbb{R})} \leq C(T), \\
\left\|\partial_{x}^{2} u(t, \cdot)\right\|_{L^{2}(\mathbb{R})}^{2}+\beta^{2} \int_{0}^{t}\left\|\partial_{x}^{5} u(s, \cdot)\right\|_{L^{2}(\mathbb{R})}^{2} d s \leq C(T),
\end{array}
$$

for every $0 \leq t \leq T$.

Proof. Let $0 \leq t \leq T$. We begin by observing that by (42),

$$
\partial_{x}^{3}\left(u^{3}\right)=6\left(\partial_{x} u\right)^{3}+18 u \partial_{x} u \partial_{x}^{2} u+3 u^{2} \partial_{x}^{3} u
$$

Multiplying (1) by $2 \partial_{x}^{4} u$, thanks to (50), an integration on $\mathbb{R}$ gives

$$
\begin{aligned}
\frac{d}{d t}\left\|\partial_{x}^{2} u(t, \cdot)\right\|_{L^{2}(\mathbb{R})}^{2}= & 2 \int_{\mathbb{R}} \partial_{x}^{4} u \partial_{t} u d x \\
= & -4 \kappa \int_{\mathbb{R}} u \partial_{x} u \partial_{x}^{4} u d x+2 \beta^{2} \int_{\mathbb{R}} \partial_{x}^{4} u \partial_{x}^{6} u d x-2 \alpha\left\|\partial_{x}^{4} u(t, \cdot)\right\|_{L^{2}(\mathbb{R})}^{2} \\
& -2 \delta^{2} \int_{\mathbb{R}} \partial_{x}^{4} u \partial_{x}^{4}\left(u^{3}\right) d x \\
= & -4 \kappa \int_{\mathbb{R}} u \partial_{x} u \partial_{x}^{4} u d x-2 \beta^{2}\left\|\partial_{x}^{5} u(t, \cdot)\right\|_{L^{2}(\mathbb{R})}^{2}-2 \alpha\left\|\partial_{x}^{4} u(t, \cdot)\right\|_{L^{2}(\mathbb{R})}^{2} \\
& +2 \delta^{2} \int_{\mathbb{R}} \partial_{x}^{5} u \partial_{x}^{3}\left(u^{3}\right) d x \\
= & -4 \kappa \int_{\mathbb{R}} u \partial_{x} u \partial_{x}^{4} u d x-2 \beta^{2}\left\|\partial_{x}^{5} u(t, \cdot)\right\|_{L^{2}(\mathbb{R})}^{2}-2 \alpha\left\|\partial_{x}^{4} u(t, \cdot)\right\|_{L^{2}(\mathbb{R})}^{2} \\
& +12 \delta^{2} \int_{\mathbb{R}}\left(\partial_{x} u\right)^{3} \partial_{x}^{5} u d x+36 \delta^{2} \int_{\mathbb{R}} u \partial_{x} u \partial_{x}^{2} u \partial_{x}^{5} u d x+6 \int_{\mathbb{R}} u^{2} \partial_{x}^{3} u \partial_{x}^{5} u d x .
\end{aligned}
$$

Therefore, we have that

$$
\begin{gathered}
\frac{d}{d t}\left\|\partial_{x}^{2} u(t, \cdot)\right\|_{L^{2}(\mathbb{R})}^{2}+2 \beta^{2}\left\|\partial_{x}^{5} u(t, \cdot)\right\|_{L^{2}(\mathbb{R})}^{2} \\
=-4 \kappa \int_{\mathbb{R}} u \partial_{x} u \partial_{x}^{4} u d x-2 \alpha\left\|\partial_{x}^{4} u(t, \cdot)\right\|_{L^{2}(\mathbb{R})}^{2}+12 \delta^{2} \int_{\mathbb{R}}\left(\partial_{x} u\right)^{3} \partial_{x}^{5} u d x \\
\quad+36 \delta^{2} \int_{\mathbb{R}} u \partial_{x} u \partial_{x}^{2} u \partial_{x}^{5} u d x+6 \int_{\mathbb{R}} u^{2} \partial_{x}^{3} u \partial_{x}^{5} u d x .
\end{gathered}
$$

Due to (22), (29) and the Young inequality,

$$
\begin{aligned}
& 4|\kappa| \int_{\mathbb{R}} u \partial_{x} u \partial_{x}^{4} u d x \leq 2 \kappa^{2} \int_{\mathbb{R}} u^{2}\left(\partial_{x} u\right)^{2} d x+2\left\|\partial_{x}^{4} u(t, \cdot)\right\|_{L^{2}(\mathbb{R})}^{2} \\
& \quad \leq 2 \kappa^{2}\|u\|_{L^{\infty}((0, T) \times \mathbb{R})}^{2}\left\|\partial_{x} u(t, \cdot)\right\|_{L^{\infty}(\mathbb{R})}^{2}+2\left\|\partial_{x}^{4} u(t, \cdot)\right\|_{L^{2}(\mathbb{R})}^{2} \\
& \quad \leq C(T)+2\left\|\partial_{x}^{4} u(t, \cdot)\right\|_{L^{2}(\mathbb{R})^{\prime}}^{2} \\
& 12 \delta^{2} \int_{\mathbb{R}}\left|\partial_{x} u\right|^{3}\left|\partial_{x}^{5}\right| u d x=2 \int_{\mathbb{R}}\left|\frac{6 \delta^{2}\left(\partial_{x} u\right)^{3} d x}{\beta \sqrt{D_{1}}}\right|\left|\beta \sqrt{D_{1}} \partial_{x}^{5} u\right| d x
\end{aligned}
$$




$$
\begin{aligned}
& \leq \frac{36 \delta^{2}}{\beta^{2} D_{1}} \int_{\mathbb{R}}\left(\partial_{x} u\right)^{6} d x+\beta^{2} D_{1}\left\|\partial_{x}^{5} u(t, \cdot)\right\|_{L^{2}(\mathbb{R})}^{2} \\
& \leq \frac{C_{0}}{D_{1}}\left\|\partial_{x} u\right\|_{L^{2}((0, T) \times \mathbb{R})}^{2}\left\|\partial_{x} u(t, \cdot)\right\|_{L^{4}(\mathbb{R})}^{4}+\beta^{2} D_{1}\left\|\partial_{x}^{5} u(t, \cdot)\right\|_{L^{2}(\mathbb{R})^{\prime}}^{2} \\
& 36 \delta^{2} \int_{\mathbb{R}}\left|u\left\|\partial_{x} u\right\| \partial_{x}^{2} u\left\|\partial_{x}^{5} u\left|d x=36 \delta^{2}\|u\|_{L^{\infty}((0, T) \times \mathbb{R})} \int_{\mathbb{R}}\right| \partial_{x} u\right\| \partial_{x}^{2} u \| \partial_{x}^{5} u\right| d x \leq 2 C(T) \int_{\mathbb{R}}\left|\partial_{x} u\left\|\partial_{x}^{2} u\right\| \partial_{x}^{5} u\right| d x=2 \int_{\mathbb{R}}\left|\frac{C(T) \partial_{x} u \partial_{x}^{2} u}{\beta \sqrt{D_{1}}}\right|\left|\beta \sqrt{D_{1}} \partial_{x}^{5} u\right| d x \\
& \leq \frac{C(T)}{D_{1}} \int_{\mathbb{R}}\left(\partial_{x} u\right)^{2}\left(\partial_{x}^{2} u\right)^{2} d x+\beta^{2} D_{1}\left\|\partial_{x}^{5} u(t, \cdot)\right\|_{L^{2}(\mathbb{R})}^{2} \\
& \leq \frac{C(T)}{D_{1}}\left\|\partial_{x} u\right\|_{L^{\infty}((0, T) \times \mathbb{R})}^{2}\left\|\partial_{x}^{2} u(t, \cdot)\right\|_{L^{2}(\mathbb{R})}^{2}+\beta^{2} D_{1}\left\|\partial_{x}^{5} u(t, \cdot)\right\|_{L^{2}(\mathbb{R})^{\prime}}^{2} \\
& 6 \delta^{2} \int_{\mathbb{R}} u^{2}\left|\partial_{x}^{3} u\left\|\partial_{x}^{5} u\left|d x=6 \delta^{2}\|u\|_{L^{\infty}((0, T) \times \mathbb{R})}^{2} \int_{\mathbb{R}}\right| \partial_{x}^{3} u\right\| \partial_{x}^{5} u\right| d x \\
& \leq 2 C(T) \int_{\mathbb{R}}\left|\partial_{x}^{3} u \| \partial_{x}^{5} u\right| d x=2 \int_{\mathbb{R}}\left|\frac{C(T) \partial_{x}^{3} u}{\beta \sqrt{D_{1}}}\right|\left|\beta \sqrt{D_{1}} \partial_{x}^{5} u\right| d x \\
& \leq \frac{C(T)}{D_{1}}\left\|\partial_{x}^{3} u(t, \cdot)\right\|_{L^{2}(\mathbb{R})}^{2}+\beta^{2}\left\|\partial_{x}^{5} u(t, \cdot)\right\|_{L^{2}(\mathbb{R})}^{2}
\end{aligned}
$$

where $D_{1}$ is a positive constant, which will specified later. It follows from (51) that

$$
\begin{aligned}
\frac{d}{d t}\left\|\partial_{x}^{2} u(t, \cdot)\right\|_{L^{2}(\mathbb{R})}^{2}+\beta^{2}\left(2-3 D_{1}\right)\left\|\partial_{x}^{5} u(t, \cdot)\right\|_{L^{2}(\mathbb{R})}^{2} \\
\leq C(T)+C_{0}\left\|\partial_{x}^{4} u(t, \cdot)\right\|_{L^{2}(\mathbb{R})}^{2}+\frac{C_{0}}{D_{1}}\left\|\partial_{x} u\right\|_{L^{2}((0, T) \times \mathbb{R})}^{2}\left\|\partial_{x} u(t, \cdot)\right\|_{L^{4}(\mathbb{R})}^{4} \\
\quad+\frac{C(T)}{D_{1}}\left\|\partial_{x} u\right\|_{L^{\infty}((0, T) \times \mathbb{R})}^{2}\left\|\partial_{x}^{2} u(t, \cdot)\right\|_{L^{2}(\mathbb{R})}^{2}+\frac{C(T)}{D_{1}}\left\|\partial_{x}^{3} u(t, \cdot)\right\|_{L^{2}(\mathbb{R})}^{2} .
\end{aligned}
$$

Taking $D_{1}=\frac{1}{3}$, we have that

$$
\begin{aligned}
\frac{d}{d t}\left\|\partial_{x}^{2} u(t, \cdot)\right\|_{L^{2}(\mathbb{R})}^{2}+\beta^{2}\left\|\partial_{x}^{5} u(t, \cdot)\right\|_{L^{2}(\mathbb{R})}^{2} \\
\leq C(T)+C_{0}\left\|\partial_{x}^{4} u(t, \cdot)\right\|_{L^{2}(\mathbb{R})}^{2}+C_{0}\left\|\partial_{x} u\right\|_{L^{2}((0, T) \times \mathbb{R})}^{2}\left\|\partial_{x} u(t, \cdot)\right\|_{L^{4}(\mathbb{R})}^{4} \\
\quad+C(T)\left\|\partial_{x} u\right\|_{L^{\infty}((0, T) \times \mathbb{R})}^{2}\left\|\partial_{x}^{2} u(t, \cdot)\right\|_{L^{2}(\mathbb{R})}^{2}+C(T)\left\|\partial_{x}^{3} u(t, \cdot)\right\|_{L^{2}(\mathbb{R})}^{2}
\end{aligned}
$$

Integrating on $(0, t)$, by (3), (23), (30), (40) and (41), we have that

$$
\begin{aligned}
\| \partial_{x}^{2} u(t, \cdot) & \left\|_{L^{2}(\mathbb{R})}^{2}+\beta^{2} \int_{0}^{t}\right\| \partial_{x}^{5} u(s, \cdot) \|_{L^{2}(\mathbb{R})}^{2} d s \\
\leq & C_{0}+C(T) t+C_{0} \int_{0}^{t}\left\|\partial_{x}^{4} u(s, \cdot)\right\|_{L^{2}(\mathbb{R})}^{2} d s+C(T) \int_{0}^{t}\left\|\partial_{x}^{3} u(s, \cdot)\right\|_{L^{2}(\mathbb{R})}^{2} d s \\
& +C_{0}\left\|\partial_{x} u\right\|_{L^{2}((0, T) \times \mathbb{R})}^{2} \int_{0}^{t}\left\|\partial_{x} u(s, \cdot)\right\|_{L^{4}(\mathbb{R})}^{4} d s \\
& +C(T)\left\|\partial_{x} u\right\|_{L^{\infty}((0, T) \times \mathbb{R})}^{2} \int_{0}^{t}\left\|\partial_{x}^{2} u(s, \cdot)\right\|_{L^{2}(\mathbb{R})}^{2} d s \\
\leq & C(T)\left(1+\left\|\partial_{x} u\right\|_{L^{2}((0, T) \times \mathbb{R})}^{2}\right) .
\end{aligned}
$$

We prove (48). Thanks to (22), (52) and the Hölder inequality,

$$
\left(\partial_{x} u(t, x)\right)^{2}=2 \int_{-\infty}^{x} \partial_{x} u \partial_{x}^{2} u d x \leq 2 \int_{\mathbb{R}}\left|\partial_{x} u \partial_{x}^{2} u\right| d x
$$




$$
\leq 2\left\|\partial_{x} u(t, \cdot)\right\|_{L^{2}(\mathbb{R})}\left\|\partial_{x}^{2} u(t, \cdot)\right\|_{L^{2}(\mathbb{R})} \leq C(T) \sqrt{1+\left\|\partial_{x} u\right\|_{L^{2}((0, T) \times \mathbb{R})}^{2}} .
$$

Therefore,

$$
\left\|\partial_{x} u\right\|_{L^{\infty}((0, T) \times \mathbb{R})}^{4}-C(T)\left\|\partial_{x} u\right\|_{L^{2}((0, T) \times \mathbb{R})}^{2}-C(T) \leq 0,
$$

which gives (48).

Finally, (49) follows from (48) and (52).

Lemma 6. Fix $T>0$. There exists a constant $C(T)>0$, such that

$$
\begin{array}{r}
\left\|\partial_{x}^{2} u\right\|_{L^{\infty}((0, T) \times \mathbb{R})} \leq C(T), \\
\left\|\partial_{x}^{3} u(t, \cdot)\right\|_{L^{2}(\mathbb{R})}^{2}+\beta^{2} \int_{0}^{t}\left\|\partial_{x}^{6} u(s, \cdot)\right\|_{L^{2}(\mathbb{R})}^{2} d s \leq C(T),
\end{array}
$$

for every $0 \leq t \leq T$.

Proof. Let $0 \leq t \leq T$. We begin by observing that by (42), we have that

$$
\partial_{x}^{4}\left(u^{3}\right)=36\left(\partial_{x} u\right)^{2} \partial_{x}^{2} u+18 u\left(\partial_{x}^{2} u\right)^{2}+6 u \partial_{x} u \partial_{x}^{3} u+3 u^{2} \partial_{x}^{4} u .
$$

Multiplying (1) by $-2 \partial_{x}^{6} u$, thanks to (56), an integration on $\mathbb{R}$ gives

$$
\begin{aligned}
\frac{d}{d t}\left\|\partial_{x}^{3} u(t, \cdot)\right\|_{L^{2}(\mathbb{R})}^{2}= & -2 \int_{\mathbb{R}} \partial_{x}^{6} u \partial_{t} u d x \\
= & 4 \kappa \int_{\mathbb{R}} u \partial_{x} u \partial_{x}^{6} u d x-2 \beta^{2}\left\|\partial_{x}^{6} u(t, \cdot)\right\|_{L^{2}(\mathbb{R})}^{2} \\
& +2 \alpha \int_{\mathbb{R}} \partial_{x}^{4} u \partial_{x}^{6} u d x+2 \delta^{2} \int_{\mathbb{R}} \partial_{x}^{6} u \partial_{x}^{2}\left(u^{3}\right) d x \\
= & -4 \kappa \int_{\mathbb{R}}\left(\partial_{x} u\right)^{2} \partial_{x}^{5} u d x-4 \kappa \int_{\mathbb{R}} u \partial_{x}^{2} u \partial_{x}^{5} u d x-2 \beta^{2}\left\|\partial_{x}^{6} u(t, \cdot)\right\|_{L^{2}(\mathbb{R})}^{2} \\
& -2 \alpha\left\|\partial_{x}^{5} u(t, \cdot)\right\|_{L^{2}(\mathbb{R})}^{2}+72 \delta^{2} \int_{\mathbb{R}}\left(\partial_{x} u\right)^{3} \partial_{x}^{2} u \partial_{x}^{6} u d x \\
& +36 \delta^{2} \int_{\mathbb{R}} u\left(\partial_{x}^{2} u\right)^{2} \partial_{x}^{6} u d x+12 \delta^{2} \int_{\mathbb{R}} u \partial_{x} u \partial_{x}^{3} u \partial_{x}^{6} u d x \\
& +6 \delta^{2} \int_{\mathbb{R}} u^{2} \partial_{x}^{4} u \partial_{x}^{6} u d x .
\end{aligned}
$$

Therefore, we have

$$
\begin{aligned}
\frac{d}{d t}\left\|\partial_{x}^{3} u(t, \cdot)\right\|_{L^{2}(\mathbb{R})}^{2}+2 \beta^{2}\left\|\partial_{x}^{6} u(t, \cdot)\right\|_{L^{2}(\mathbb{R})}^{2} & -4 \kappa \int_{\mathbb{R}}\left(\partial_{x} u\right)^{2} \partial_{x}^{5} u d x-4 \kappa \int_{\mathbb{R}} u \partial_{x}^{2} u \partial_{x}^{5} u d x-2 \alpha\left\|\partial_{x}^{5} u(t, \cdot)\right\|_{L^{2}(\mathbb{R})}^{2} \\
& +72 \delta^{2} \int_{\mathbb{R}}\left(\partial_{x} u\right)^{3} \partial_{x}^{2} u \partial_{x}^{6} u d x+36 \delta^{2} \int_{\mathbb{R}} u\left(\partial_{x}^{2} u\right)^{2} \partial_{x}^{6} u d x \\
& +12 \delta^{2} \int_{\mathbb{R}} u \partial_{x} u \partial_{x}^{3} u \partial_{x}^{6} u d x+6 \delta^{2} \int_{\mathbb{R}} u^{2} \partial_{x}^{4} u \partial_{x}^{6} u d x
\end{aligned}
$$

Due to (22), (29), (48), (49) and the Young inequality,

$$
\begin{gathered}
4|\mathcal{k}| \int_{\mathbb{R}}\left(\partial_{x} u\right)^{2}\left|\partial_{x}^{5} u\right| d x \leq 4|\mathcal{K}|\left\|\partial_{x} u\right\|_{L^{\infty}((0, T) \times \mathbb{R})} \int_{\mathbb{R}}\left|\partial_{x} u\right|\left|\partial_{x}^{5} u\right| d x \\
\leq C(T)\left\|\partial_{x} u(t, \cdot)\right\|_{L^{2}(\mathbb{R})}^{2}+C(T)\left\|\partial_{x}^{5} u(t, \cdot)\right\|_{L^{2}(\mathbb{R})}^{\prime}
\end{gathered}
$$




$$
\begin{aligned}
& \leq C(T)+C(T)\left\|\partial_{x}^{5} u(t, \cdot)\right\|_{L^{2}(\mathbb{R})}^{2}, \\
& 4|\kappa| \int_{\mathbb{R}}\left|u\left\|\partial_{x}^{2} u|| \partial_{x}^{5} u\left|d x \leq 4 \kappa\|u\|_{L^{\infty}((0, T) \times \mathbb{R})} \int_{\mathbb{R}}\right| \partial_{x}^{2} u\right\| \partial_{x}^{5} u\right| d x \\
& \leq C(T)\left\|\partial_{x}^{2} u(t, \cdot)\right\|_{L^{2}(\mathbb{R})}^{2}+C(T)\left\|\partial_{x}^{5} u(t, \cdot)\right\|_{L^{2}(\mathbb{R})}^{2} \\
& \leq C(T)+C(T)\left\|\partial_{x}^{5} u(t, \cdot)\right\|_{L^{2}(\mathbb{R})}^{2}, \\
& 72 \delta^{2} \int_{\mathbb{R}}\left|\partial_{x} u\right|^{3}\left|\partial_{x}^{2} u\left\|\partial_{x}^{6} u\left|d x \leq 72 \delta^{2}\left\|\partial_{x} u\right\|_{L^{\infty}((0, T) \times \mathbb{R})}^{3} \int_{\mathbb{R}}\right| \partial_{x}^{2} u\right\| \partial_{x}^{6} u\right| d x \\
& \leq 2 C(T) \int_{\mathbb{R}}\left|\partial_{x}^{2} u \| \partial_{x}^{6} u\right| d x \leq 2 \int_{\mathbb{R}}\left|\frac{C(T) \partial_{x}^{2} u}{\beta \sqrt{D_{2}}}\right|\left|\beta \sqrt{D_{2}} \partial_{x}^{6} u d x\right| d x \\
& \leq \frac{C(T)}{D_{2}}\left\|\partial_{x}^{2} u(t, \cdot)\right\|_{L^{2}(\mathbb{R})}^{2}+\beta^{2} D_{2}\left\|\partial_{x}^{6} u(t, \cdot)\right\|_{L^{2}(\mathbb{R})}^{2} \\
& \leq \frac{C(T)}{D_{2}}+\beta^{2} D_{2}\left\|\partial_{x}^{6} u(t, \cdot)\right\|_{L^{2}(\mathbb{R})}^{2}, \\
& 36 \delta^{2} \int_{\mathbb{R}}|u|\left(\partial_{x}^{2} u\right)^{2}\left|\partial_{x}^{6} u\right| d x \leq 36 \delta^{2}\|u\|_{L^{\infty}((0, T) \times \mathbb{R})} \int_{\mathbb{R}}\left(\partial_{x}^{2} u\right)^{2}\left|\partial_{x}^{6} u\right| d x \\
& \leq 2 C(T) \int_{\mathbb{R}}\left(\partial_{x}^{2} u\right)^{2}\left|\partial_{x}^{6} u\right| d x=2 \int_{\mathbb{R}}\left|\frac{C(T)\left(\partial_{x}^{2} u\right)^{2}}{\beta \sqrt{D_{2}}}\right|\left|\beta \sqrt{D_{2}} \partial_{x}^{6} u\right| d x \\
& \leq \frac{C(T)}{D_{2}} \int_{\mathbb{R}}\left(\partial_{x}^{2} u\right)^{4} d x+\beta^{2} D_{2}\left\|\partial_{x}^{6} u(t, \cdot)\right\|_{L^{2}(\mathbb{R})}^{2} \\
& \leq \frac{C(T)}{D_{2}}\left\|\partial_{x}^{2} u\right\|_{L^{\infty}((0, T) \times \mathbb{R})}^{2}\left\|\partial_{x}^{2} u(t, \cdot)\right\|_{L^{2}(\mathbb{R})}^{2}+\beta^{2} D_{2}\left\|\partial_{x}^{6} u(t, \cdot)\right\|_{L^{2}(\mathbb{R})}^{2}, \\
& 12 \delta^{2} \int_{\mathbb{R}}\left|u\left\|\partial_{x} u\right\| \partial_{x}^{3} u\left\|\partial_{x}^{6} u\left|d x=12 \delta^{2}\|u\|_{L^{\infty}((0, T) \times \mathbb{R})} \int_{\mathbb{R}}\right| \partial_{x} u\right\| \partial_{x}^{3} u \| \partial_{x}^{6} u\right| d x \\
& \leq 2 C(T) \int_{\mathbb{R}}\left|\partial_{x} u\left\|\partial_{x}^{3} u\right\| \partial_{x}^{6} u\right| d x \leq 2 C(T)\left\|\partial_{x} u\right\|_{L^{\infty}((0, T) \times \mathbb{R})} \int_{\mathbb{R}}\left|\partial_{x}^{3} u \| \partial_{x}^{6} u\right| d x \\
& \leq 2 C(T) \int_{\mathbb{R}}\left|\partial_{x}^{3} u\right|\left|\partial_{x}^{6} u\right| d x=2 \int_{\mathbb{R}}\left|\frac{C(T) \partial_{x}^{3} u}{\beta \sqrt{D_{2}}}\right|\left|\beta \sqrt{D_{2}} \partial_{x}^{6} u\right| d x \\
& \leq \frac{C(T)}{D_{2}}\left\|\partial_{x}^{3} u(t, \cdot)\right\|_{L^{2}(\mathbb{R})}^{2}+\beta^{2} D_{2}\left\|\partial_{x}^{6} u(t, \cdot)\right\|_{L^{2}(\mathbb{R})}^{2}, \\
& 6 \delta^{2} \int_{\mathbb{R}} u^{2}\left|\partial_{x}^{4} u\left\|\partial_{x}^{6} u\left|d x \leq 6 \delta^{2}\|u\|_{L^{\infty}((0, T) \times \mathbb{R})}^{2} \int_{\mathbb{R}}\right| \partial_{x}^{4} u\right\| \partial_{x}^{6} u\right| d x \\
& \leq 2 C(T) \int_{\mathbb{R}}\left|\partial_{x}^{4} u\right|\left|\partial_{x}^{6} u\right| d x=2 \int_{\mathbb{R}}\left|\frac{C(T) \partial_{x}^{4} u \mid}{\beta \sqrt{D_{2}}}\right|\left|\beta \sqrt{D_{2}} \partial_{x}^{6} u\right| d x \\
& \leq \frac{C(T)}{D_{2}}\left\|\partial_{x}^{4} u(t, \cdot)\right\|_{L^{2}(\mathbb{R})}^{2}+\beta^{2} D_{2}\left\|\partial_{x}^{6} u(t, \cdot)\right\|_{L^{2}(\mathbb{R})}^{2},
\end{aligned}
$$

where $D_{2}$ is a positive constant which will specified later. It follows from (57) that

$$
\begin{aligned}
\frac{d}{d t}\left\|\partial_{x}^{3} u(t, \cdot)\right\|_{L^{2}(\mathbb{R})}^{2}+2 \beta^{2}\left(1-2 D_{2}\right)\left\|\partial_{x}^{6} u(t, \cdot)\right\|_{L^{2}(\mathbb{R})}^{2} \\
\leq C(T)\left(1+\frac{1}{D_{2}}\right)+C(T)\left\|\partial_{x}^{5} u(t, \cdot)\right\|_{L^{2}(\mathbb{R})}^{2} \\
\quad+\frac{C(T)}{D_{2}}\left\|\partial_{x}^{2} u\right\|_{L^{\infty}((0, T) \times \mathbb{R})}^{2}\left\|\partial_{x}^{2} u(t, \cdot)\right\|_{L^{2}(\mathbb{R})}^{2}+\frac{C(T)}{D_{2}}\left\|\partial_{x}^{3} u(t, \cdot)\right\|_{L^{2}(\mathbb{R})}^{2} \\
\quad+\frac{C(T)}{D_{2}}\left\|\partial_{x}^{4} u(t, \cdot)\right\|_{L^{2}(\mathbb{R})}^{2} .
\end{aligned}
$$


Taking $D_{2}=\frac{1}{2}$, we have that

$$
\begin{aligned}
\frac{d}{d t}\left\|\partial_{x}^{3} u(t, \cdot)\right\|_{L^{2}(\mathbb{R})}^{2}+\beta^{2}\left\|\partial_{x}^{6} u(t, \cdot)\right\|_{L^{2}(\mathbb{R})}^{2} & \\
\leq & C(T)+C(T)\left\|\partial_{x}^{5} u(t, \cdot)\right\|_{L^{2}(\mathbb{R})}^{2}+C(T)\left\|\partial_{x}^{2} u\right\|_{L^{\infty}((0, T) \times \mathbb{R})}^{2}\left\|\partial_{x}^{2} u(t, \cdot)\right\|_{L^{2}(\mathbb{R})}^{2} \\
& +C(T)\left\|\partial_{x}^{3} u(t, \cdot)\right\|_{L^{2}(\mathbb{R})}^{2}+C(T)\left\|\partial_{x}^{4} u(t, \cdot)\right\|_{L^{2}(\mathbb{R})}^{2} \cdot
\end{aligned}
$$

(3), (23), (40), (41), (49) and an integration on (0,t) give

$$
\begin{aligned}
\frac{d}{d t}\left\|\partial_{x}^{3} u(t, \cdot)\right\|_{L^{2}(\mathbb{R})}^{2}+\beta^{2} \int_{0}^{t}\left\|\partial_{x}^{6} u(s, \cdot)\right\|_{L^{2}(\mathbb{R})}^{2} d s \\
\leq C_{0}+C(T) t+C(T) \int_{0}^{t}\left\|\partial_{x}^{5} u(s, \cdot)\right\|_{L^{2}(\mathbb{R})}^{2} d s \\
\quad+C(T) \int_{0}^{t}\left\|\partial_{x}^{3} u(s, \cdot)\right\|_{L^{2}(\mathbb{R})}^{2} d s+C(T) \int_{0}^{t}\left\|\partial_{x}^{4} u(s, \cdot)\right\|_{L^{2}(\mathbb{R})}^{2} d s \\
\quad+C(T)\left\|\partial_{x}^{2} u\right\|_{L^{\infty}((0, T) \times \mathbb{R})}^{2} \int_{0}^{t}\left\|\partial_{x}^{2} u(s, \cdot)\right\|_{L^{2}(\mathbb{R})}^{2} d s \\
\leq C(T)\left(1+\left\|\partial_{x}^{2} u\right\|_{L^{\infty}((0, T) \times \mathbb{R})}^{2}\right) .
\end{aligned}
$$

We prove (54). Thanks to (49), (58) the Hölder inequality,

$$
\begin{aligned}
\left(\partial_{x}^{2} u(t, x)\right)^{2} & =2 \int_{-\infty}^{x} \partial_{x}^{2} u \partial_{x}^{3} u d y \leq 2 \int_{\mathbb{R}}\left|\partial_{x}^{2} u \| \partial_{x}^{3} u\right| d x \\
& \leq 2\left\|\partial_{x}^{2} u(t, \cdot)\right\|_{L^{2}(\mathbb{R})}\left\|\partial_{x}^{3} u(t, \cdot)\right\|_{L^{2}(\mathbb{R})} \leq C(T) \sqrt{\left(1+\left\|\partial_{x}^{2} u\right\|_{L^{\infty}((0, T) \times \mathbb{R})}^{2}\right)} .
\end{aligned}
$$

Therefore,

$$
\left\|\partial_{x}^{2} u\right\|_{L^{\infty}((0, T) \times \mathbb{R})}^{4}-C(T)\left\|\partial_{x}^{2} u\right\|_{L^{\infty}((0, T) \times \mathbb{R})}^{2}-C(T) \leq 0,
$$

which gives (54).

Finally, (55) follows from (54) and (58).

Lemma 7. Fix $T>0$. There exists a constant $C(T)>0$, such that

$$
\beta^{2}\left\|\partial_{x}^{3} u(t, \cdot)\right\|_{L^{2}(\mathbb{R})}^{2}+\int_{0}^{t}\left\|\partial_{t} u(s, \cdot)\right\|_{L^{2}(\mathbb{R})}^{2} d s \leq C(T),
$$

for every $0 \leq t \leq T$.

Proof. Let $0 \leq t \leq T$. Multiplying (1) by $2 \partial_{t} u$, we have

$$
2\left(\partial_{t} u\right)+4 \kappa u \partial_{x} u \partial_{t} u-2 \beta^{2} \partial_{x}^{6} u \partial_{t} u+2 \alpha \partial_{x}^{4} u+2 \delta^{2} \partial_{t} u \partial_{x}^{4}\left(u^{3}\right) .
$$

Since

$$
\begin{aligned}
-2 \beta^{2} \int_{\mathbb{R}} \partial_{x}^{6} u \partial_{t} u d x & =\beta^{2} \frac{d}{d t}\left\|\partial_{x}^{3} u(t, \cdot)\right\|_{L^{2}(\mathbb{R})}^{2}, \\
2 \alpha \int_{\mathbb{R}} \partial_{x}^{4} u \partial_{t} u d x & =\alpha \frac{d}{d t}\left\|\partial_{x}^{2} u(t, \cdot)\right\|_{L^{2}(\mathbb{R})}^{2},
\end{aligned}
$$


thanks to (56), an integration of (60) on $\mathbb{R}$ gives

$$
\begin{aligned}
\frac{d}{d t}\left(\beta^{2}\left\|\partial_{x}^{3} u(t, \cdot)\right\|_{L^{2}(\mathbb{R})}^{2}+\alpha\left\|\partial_{x}^{2} u(t, \cdot)\right\|_{L^{2}(\mathbb{R})}^{2}\right)+2\left\|\partial_{t} u(t, \cdot)\right\|_{L^{2}(\mathbb{R})}^{2} \\
=-4 \kappa \int_{\mathbb{R}} u \partial_{x} u \partial_{t} u d x-2 \delta^{2} \int_{\mathbb{R}} \partial_{t} u \partial_{x}^{4}\left(u^{3}\right) d x \\
=-4 \kappa \int_{\mathbb{R}} u \partial_{x} u \partial_{t} u d x-72 \delta^{2} \int_{\mathbb{R}}\left(\partial_{x} u\right)^{2} \partial_{x}^{2} u \partial_{t} u d x-36 \delta^{2} \int_{\mathbb{R}} u\left(\partial_{x}^{2} u\right)^{2} \partial_{t} u d x \\
\quad-12 \delta^{2} \int_{\mathbb{R}} u \partial_{x} u \partial_{x}^{3} u \partial_{t} u d x-6 \delta^{2} \int_{\mathbb{R}} u^{2} \partial_{x}^{4} u \partial_{t} u d x .
\end{aligned}
$$

Due to (22), (29), (48), (49), (54), (55) and the Young inequality,

$$
\begin{aligned}
& 4|\mathcal{}| \int_{\mathbb{R}}|u|\left|\partial_{x} u\right||| \partial_{t} u|d x=4| \kappa\left|\|u\|_{L^{\infty}((0, T) \times \mathbb{R})} \int_{\mathbb{R}}\right| \partial_{x} u \| \partial_{t} u \mid d x \\
& \leq 2 C(T) \int_{\mathbb{R}}\left|\partial_{x} u\right|\left|\partial_{t} u\right| d x \leq 2 \int_{\mathbb{R}}\left|\frac{C(T) \partial_{x} u}{\sqrt{D_{3}}}\right|\left|\sqrt{D_{3}} \partial_{t} u\right| d x \\
& \leq \frac{C(T)}{D_{3}}\left\|\partial_{x} u(t, \cdot)\right\|_{L^{2}(\mathbb{R})}^{2}+D_{3}\left\|\partial_{t} u(t, \cdot)\right\|_{L^{2}(\mathbb{R})}^{2} \\
& \leq \frac{C(T)}{D_{3}}+D_{3}\left\|\partial_{t} u(t, \cdot)\right\|_{L^{2}(\mathbb{R})}^{2} ， \\
& 72 \delta^{2} \int_{\mathbb{R}}\left(\partial_{x} u\right)^{2}\left|\partial_{x}^{2} u\left\|\partial_{t} u\left|d x \leq 72 \delta^{2}\left\|\partial_{x} u\right\|_{L^{\infty}((0, T) \times \mathbb{R})}^{2} \int_{\mathbb{R}}\right| \partial_{x}^{2} u\right\| \partial_{t} u\right| d x \\
& \leq 2 C(T) \int_{\mathbb{R}}\left|\partial_{x}^{2} u\right|\left|\partial_{t} u\right| d x \leq 2 \int_{\mathbb{R}}\left|\frac{C(T) \partial_{x}^{2} u}{\sqrt{D_{3}}}\right|\left|\sqrt{D_{3}} \partial_{t} u\right| d x \\
& \leq \frac{C(T)}{D_{3}}\left\|\partial_{x}^{2} u(t, \cdot)\right\|_{L^{2}(\mathbb{R})}^{2}+D_{3}\left\|\partial_{t} u(t, \cdot)\right\|_{L^{2}(\mathbb{R})}^{2} \\
& \leq \frac{C(T)}{D_{3}}+D_{3}\left\|\partial_{t} u(t, \cdot)\right\|_{L^{2}(\mathbb{R})}^{2} ， \\
& 36 \delta^{2} \int_{\mathbb{R}}|u|\left(\partial_{x}^{2} u\right)^{2}\left|\partial_{t} u\right| d x \leq 36 \delta^{2}\|u\|_{L^{\infty}((0, T) \times \mathbb{R})} \int_{\mathbb{R}}\left(\partial_{x}^{2} u^{2}\right)\left|\partial_{t} u\right| d x \\
& \leq 2 C(T) \int_{\mathbb{R}}\left(\partial_{x}^{2} u\right)^{2}\left|\partial_{t} u\right| d x \leq 2 C(T)\left\|\partial_{x}^{2} u\right\|_{L^{\infty}((0, T) \times \mathbb{R})} \int_{\mathbb{R}}\left|\partial_{x}^{2} u \| \partial_{t} u\right| d x \\
& \leq 2 C(T) \int_{\mathbb{R}}\left|\partial_{x}^{2} u\right|\left|\partial_{t} u\right| d x=2 \int_{\mathbb{R}}\left|\frac{C(T) \partial_{x}^{2} u}{\sqrt{D_{3}}}\right|\left|\sqrt{D_{3}} \partial_{t} u\right| d x \\
& \leq \frac{C(T)}{D_{3}}\left\|\partial_{x}^{2} u(t, \cdot)\right\|_{L^{2}(\mathbb{R})}^{2}+D_{3}\left\|\partial_{t} u(t, \cdot)\right\|_{L^{2}(\mathbb{R})}^{2} \\
& \leq \frac{C(T)}{D_{3}}+D_{3}\left\|\partial_{t} u(t, \cdot)\right\|_{L^{2}(\mathbb{R})}^{2} ， \\
& 12 \delta^{2} \int_{\mathbb{R}}\left|u\left\|\partial_{x} u\right\| \partial_{x}^{3} u\left\|\partial_{t} u\left|d x \leq 12 \delta^{2}\|u\|_{L^{\infty}((0, T) \times \mathbb{R})} \int_{\mathbb{R}}\right| \partial_{x} u\right\| \partial_{x}^{3} u \| \partial_{t} u\right| d x \\
& \leq 2 C(T) \int_{\mathbb{R}} \int_{\mathbb{R}}\left|\partial_{x} u\left\|\partial_{x}^{3} u\right\| \partial_{t} u\right| d x \leq 2 C(T)\left\|\partial_{x} u\right\|_{L^{\infty}((0, T) \times \mathbb{R})} \int_{\mathbb{R}}\left|\partial_{x}^{3} u \| \partial_{t} u\right| d x \\
& \leq 2 C(T) \int_{\mathbb{R}}\left|\partial_{x}^{3} u\right|\left|\partial_{t} u\right| d x \leq 2 \int_{\mathbb{R}}\left|\frac{C(T) \partial_{x}^{3} u}{\sqrt{D_{3}}}\right|\left|\sqrt{D_{3}} \partial_{t} u\right| d x \\
& \leq \frac{C(T)}{D_{3}}\left\|\partial_{x}^{3} u(t, \cdot)\right\|_{L^{2}(\mathbb{R})}^{2}+D_{3}\left\|\partial_{t} u(t, \cdot)\right\|_{L^{2}(\mathbb{R})}^{2} \\
& \leq \frac{C(T)}{D_{3}}+D_{3}\left\|\partial_{t} u(t, \cdot)\right\|_{L^{2}(\mathbb{R})}^{2} ， \\
& 6 \delta^{2} \int_{\mathbb{R}} u^{2}\left|\partial_{x}^{4} u\left\|\partial_{t} u\left|d x \leq 6 \delta^{2}\|u\|_{L^{\infty}((0, T) \times \mathbb{R})}^{2} \int_{\mathbb{R}}\right| \partial_{x}^{4} u\right\| \partial_{t} u\right| d x
\end{aligned}
$$




$$
\begin{aligned}
& \leq 2 C(T) \int_{\mathbb{R}} \int_{\mathbb{R}}\left|\partial_{x}^{4} u\right|\left|\partial_{t} u\right| d x \leq 2 \int_{\mathbb{R}}\left|\frac{C(T) \partial_{x}^{4} u}{\sqrt{D_{3}}}\right|\left|\sqrt{D_{3}} \partial_{t} u\right| d x \\
& \leq \frac{C(T)}{D_{3}}\left\|\partial_{x}^{4} u(t, \cdot)\right\|_{L^{2}(\mathbb{R})}^{2}+D_{3}\left\|\partial_{t} u(t, \cdot)\right\|_{L^{2}(\mathbb{R})}^{2},
\end{aligned}
$$

where $D_{3}$ is a positive constant, which will specified later. Therefore, by (61),

$$
\begin{aligned}
& \frac{d}{d t}\left(\beta^{2}\left\|\partial_{x}^{3} u(t, \cdot)\right\|_{L^{2}(\mathbb{R})}^{2}+\alpha\left\|\partial_{x}^{2} u(t, \cdot)\right\|_{L^{2}(\mathbb{R})}^{2}\right)+\left(2-5 D_{3}\right)\left\|\partial_{t} u(t, \cdot)\right\|_{L^{2}(\mathbb{R})}^{2} \\
& \quad \leq \frac{C(T)}{D_{3}}+\frac{C(T)}{D_{3}}\left\|\partial_{x}^{4} u(t, \cdot)\right\|_{L^{2}(\mathbb{R})}^{2} .
\end{aligned}
$$

Taking $D_{3}=\frac{1}{5}$, we have that

$$
\begin{aligned}
& \frac{d}{d t}\left(\beta^{2}\left\|\partial_{x}^{3} u(t, \cdot)\right\|_{L^{2}(\mathbb{R})}^{2}+\alpha\left\|\partial_{x}^{2} u(t, \cdot)\right\|_{L^{2}(\mathbb{R})}^{2}\right)+\left\|\partial_{t} u(t, \cdot)\right\|_{L^{2}(\mathbb{R})}^{2} \\
& \leq C(T)+C(T)\left\|\partial_{x}^{4} u(t, \cdot)\right\|_{L^{2}(\mathbb{R})}^{2} \cdot
\end{aligned}
$$

It follows from (3), (41) and an integration on $(0, t)$ that

$$
\begin{gathered}
\beta^{2}\left\|\partial_{x}^{3} u(t, \cdot)\right\|_{L^{2}(\mathbb{R})}^{2}+\alpha\left\|\partial_{x}^{2} u(t, \cdot)\right\|_{L^{2}(\mathbb{R})}^{2}+\int_{0}^{t}\left\|\partial_{t} u(s, \cdot)\right\|_{L^{2}(\mathbb{R})}^{2} d s \\
\leq C_{0}+C(T) t+C(T) \int_{0}^{t}\left\|\partial_{x}^{4} u(s, \cdot)\right\|_{L^{2}(\mathbb{R})}^{2} d s \leq C(T) .
\end{gathered}
$$

Therefore, by (49),

$$
\begin{aligned}
\beta^{2}\left\|\partial_{x}^{3} u(t, \cdot)\right\|_{L^{2}(\mathbb{R})}^{2} & +\int_{0}^{t}\left\|\partial_{t} u(s, \cdot)\right\|_{L^{2}(\mathbb{R})}^{2} d s \\
\leq & C(T)-\alpha\left\|\partial_{x}^{2} u(t, \cdot)\right\|_{L^{2}(\mathbb{R})}^{2} \\
\leq & C(T)+|\alpha|\left\|\partial_{x}^{2} u(t, \cdot)\right\|_{L^{2}(\mathbb{R})}^{2} \leq C(T),
\end{aligned}
$$

which gives (59).

Lemma 8. Fix $T>0$. There exists a constant $C(T)>0$, such that

$$
\int_{0}^{t}\left\|\partial_{t} P(s, \cdot)\right\|_{L^{2}(\mathbb{R})}^{2} d s \leq C(T)
$$

for every $0 \leq t \leq T$.

Proof. Let $0 \leq t \leq T$. Multiplying (18) by $2 \partial_{t} P$, thanks to (42), an integration on $\mathbb{R}$ gives

$$
\begin{aligned}
2\left\|\partial_{t} P(t, \cdot)\right\|_{L^{2}(\mathbb{R})}^{2}= & -4 \kappa \int_{\mathbb{R}} u^{2} \partial_{t} P d x+2 \beta^{2} \int_{\mathbb{R}} \partial_{x}^{5} u \partial_{t} P d x-2 \alpha \int_{\mathbb{R}} \partial_{x}^{3} u \partial_{t} P d x \\
& -12 \delta^{2} \int_{\mathbb{R}}\left(\partial_{x} u\right)^{3} \partial_{t} P d x-36 \delta^{2} \int_{\mathbb{R}} u \partial_{x} u \partial_{x}^{2} u \partial_{t} P d x \\
& -6 \delta^{2} \int_{\mathbb{R}} u^{2} \partial_{x}^{3} u \partial_{t} P d x
\end{aligned}
$$


Due to (22), (29), (48), (49), (55) and the Young inequality,

$$
\begin{aligned}
& 4|\kappa| \int_{\mathbb{R}} u^{2}\left|\partial_{t} P\right| d x=2 \int_{\mathbb{R}}\left|\frac{2 \kappa u^{2}}{\sqrt{D_{4}}}\right|\left|\sqrt{D_{4}} \partial_{t} P\right| d x \\
& \leq \frac{4 \kappa^{2}}{D_{4}}\|u(t, \cdot)\|_{L^{4}(\mathbb{R})}^{4}+D_{4}\left\|\partial_{t} P(t, \cdot)\right\|_{L^{2}(\mathbb{R})}^{2} \\
& \leq \frac{C(T)}{D_{4}}\|u(t, \cdot)\|_{L^{4}(\mathbb{R})}^{4}+D_{4}\left\|\partial_{t} P(t, \cdot)\right\|_{L^{2}(\mathbb{R})}^{2} \\
& \leq \frac{C(T)}{D_{4}}+D_{4}\left\|\partial_{t} P(t, \cdot)\right\|_{L^{2}(\mathbb{R})}^{2} ， \\
& +2 \beta^{2} \int_{\mathbb{R}}\left|\partial_{x}^{5} u\right|\left|\partial_{t} P\right| d x=2 \int_{\mathbb{R}}\left|\frac{\beta^{2} \partial_{x}^{5} u}{\sqrt{D_{4}}}\right|\left|\sqrt{D_{4}} \partial_{t} P\right| d x \\
& \leq \frac{\beta^{4}}{D_{4}}\left\|\partial_{x}^{5} u(t, \cdot)\right\|_{L^{2}(\mathbb{R})}^{2}+D_{4}\left\|\partial_{t} P(t, \cdot)\right\|_{L^{2}(\mathbb{R})}^{2} ， \\
& 2|\alpha| \int_{\mathbb{R}}\left|\partial_{x}^{3} u\right|\left|\partial_{t} P\right| d x=2 \int_{\mathbb{R}}\left|\frac{\alpha \partial_{x}^{3} u}{\sqrt{D_{4}}}\right|\left|\sqrt{D_{4}} \partial_{t} P\right| d x \\
& \leq \frac{\alpha^{2}}{D_{4}}\left\|\partial_{x}^{3} u(t, \cdot)\right\|_{L^{2}(\mathbb{R})}^{2}+D_{4}\left\|\partial_{t} P(t, \cdot)\right\|_{L^{2}(\mathbb{R})}^{2} \\
& \leq \frac{C(T)}{D_{4}}\left\|\partial_{x}^{3} u(t, \cdot)\right\|_{L^{2}(\mathbb{R})}^{2}+D_{4}\left\|\partial_{t} P(t, \cdot)\right\|_{L^{2}(\mathbb{R})}^{2} \\
& \leq \frac{C(T)}{D_{4}}+D_{4}\left\|\partial_{t} P(t, \cdot)\right\|_{L^{2}(\mathbb{R})}^{2} ， \\
& 12 \delta^{2} \int_{\mathbb{R}}\left|\partial_{x} u\right|^{3}\left|\partial_{t} P\right| d x=12 \delta^{2}\left\|\partial_{x} u\right\|_{L^{\infty}((0, T) \times \mathbb{R})}^{2} \int_{\mathbb{R}}\left|\partial_{x} u \| \partial_{t} P\right| d x \\
& \leq 2 C(T) \int_{\mathbb{R}}\left|\partial_{x} u \| \partial_{t} P\right| d x=2 \int_{\mathbb{R}}\left|\frac{C(T) \partial_{x} u}{\sqrt{D_{4}}}\right|\left|\sqrt{D_{4}} \partial_{t} P\right| d x \\
& \leq \frac{C(T)}{D_{4}}\left\|\partial_{x} u(t, \cdot)\right\|_{L^{2}(\mathbb{R})}^{2}+D_{4}\left\|\partial_{t} P(t, \cdot)\right\|_{L^{2}(\mathbb{R})}^{2} \\
& \leq \frac{C(T)}{D_{4}}+D_{4}\left\|\partial_{t} P(t, \cdot)\right\|_{L^{2}(\mathbb{R})}^{2}, \\
& 36 \delta^{2} \int_{\mathbb{R}}\left|u\left\|\partial_{x} u\right\| \partial_{x}^{2} u\left\|\partial_{t} P\left|d x \leq 36 \delta^{2}\|u\|_{L^{\infty}((0, T) \times \mathbb{R})} \int_{\mathbb{R}}\right| \partial_{x} u\right\| \partial_{x}^{2} u \| \partial_{t} P\right| d x \\
& \leq 2 C(T) \int_{\mathbb{R}}\left|\partial_{x} u\left\|\partial_{x}^{2} u\right\| \partial_{t} P\right| d x \leq 2 C(T)\left\|\partial_{x} u\right\|_{L^{\infty}((0, T) \times \mathbb{R})} \int_{\mathbb{R}}\left|\partial_{x}^{2} u \| \partial_{t} P\right| d x \\
& \leq 2 C(T) \int_{\mathbb{R}}\left|\partial_{x}^{2} u \| \partial_{t} P\right| d x=2 \int_{\mathbb{R}}\left|\frac{C(T) \partial_{x}^{2} u}{\sqrt{D_{4}}}\right|\left|\sqrt{D_{4}} \partial_{t} P\right| d x \\
& \leq \frac{C(T)}{D_{4}}\left\|\partial_{x}^{2} u(t, \cdot)\right\|_{L^{2}(\mathbb{R})}^{2}+D_{4}\left\|\partial_{t} P(t, \cdot)\right\|_{L^{2}(\mathbb{R})}^{2} \\
& \leq \frac{C(T)}{D_{4}}+D_{4}\left\|\partial_{t} P(t, \cdot)\right\|_{L^{2}(\mathbb{R})}^{2} ， \\
& 6 \delta^{2} \int_{\mathbb{R}} u^{2}\left|\partial _ { x } ^ { 3 } u \left\|\partial_{t} P\left|d x \leq 6 \delta^{2}\|u\|_{L^{\infty}((0, T) \times \mathbb{R})}^{2} \int_{\mathbb{R}}\right| \partial_{x}^{3} u|| \partial_{t} P \mid d x\right.\right. \\
& \leq 2 C(T) \int_{\mathbb{R}}\left|\partial_{x}^{3} u\right|\left|\partial_{t} P\right| d x=2 \int_{\mathbb{R}}\left|\frac{C(T) \partial_{x}^{2} u}{D_{4}}\right|\left|D_{4} \partial_{t} P\right| d x \\
& \leq \frac{C(T)}{D_{4}}\left\|\partial_{x}^{3} u(t, \cdot)\right\|_{L^{2}(\mathbb{R})}^{2}+D_{4}\left\|\partial_{t} P(t, \cdot)\right\|_{L^{2}(\mathbb{R})}^{2} \\
& \leq \frac{C(T)}{D_{4}}+D_{4}\left\|\partial_{t} P(t, \cdot)\right\|_{L^{2}(\mathbb{R})}^{2} .
\end{aligned}
$$


It follows from (63) that

$$
2\left(1-3 D_{6}\right)\left\|\partial_{t} P(t, \cdot)\right\|_{L^{2}(\mathbb{R})}^{2} \leq \frac{C(T)}{D_{4}}+\frac{\beta^{2}}{D_{4}}\left\|\partial_{x}^{5} u(t, \cdot)\right\|_{L^{2}(\mathbb{R})}^{2} .
$$

Taking $D_{4}=\frac{1}{6}$, we have that

$$
\left\|\partial_{t} P(t, \cdot)\right\|_{L^{2}(\mathbb{R})}^{2} \leq C(T)+6 \beta^{4}\left\|\partial_{x}^{5} u(t, \cdot)\right\|_{L^{2}(\mathbb{R})}^{2} .
$$

Integrating on $(0, t)$, by (49), we obtain

$$
\int_{0}^{t}\left\|\partial_{t} P(s, \cdot)\right\|_{L^{2}(\mathbb{R})} d s \leq C(T) t+6 \beta^{4} \int_{0}^{t}\left\|\partial_{x}^{5} u(s, \cdot)\right\|_{L^{2}(\mathbb{R})}^{2} d s \leq C(T),
$$

which gives (62).

\section{Proof of Theorem 1}

This section devoted to the proof of Theorem 1.

Proof of Theorem 1. Fix $T>0$. Thanks to Lemmas 3, 4, 5, 6, (7) and the Cauchy-Kovalevskaya Theorem [67], we have that $u$ is solution of (1) and (8) holds. In particular, by Lemma 1, we get (9). Moreover, by Lemmas 2, 3, 4, 5, 6, 8 and Remark 1, we have that

$$
P \in H^{1}((0, T) \times \mathbb{R}) \cap L^{\infty}\left(0, T ; H^{4}(\mathbb{R})\right) .
$$

We prove (10). Let $u_{1}$ and $u_{2}$ be two solutions of (1), which verify (8), which is

$$
\begin{aligned}
& \begin{cases}\partial_{t} u_{1}+\kappa \partial_{x} u_{1}^{2}-\beta^{2} \partial_{x}^{6} u_{1}+\alpha \partial_{x}^{4} u_{1}+\delta^{2} \partial_{x}^{4}\left(u_{1}^{3}\right)=0, & t>0, x \in \mathbb{R}, \\
u_{1}(0, x)=u_{1,0}(x), & x \in \mathbb{R},\end{cases} \\
& \begin{cases}\partial_{t} u_{2}+\kappa \partial_{x} u_{2}^{2}-\beta^{2} \partial_{x}^{6} u_{2}+\alpha \partial_{x}^{4} u_{2}+\delta^{2} \partial_{x}^{4}\left(u_{2}^{3}\right)=0, & t>0, x \in \mathbb{R}, \\
u_{2}(0, x)=u_{2,0}(x), & x \in \mathbb{R},\end{cases}
\end{aligned}
$$

Then, the function

$$
\omega=u_{1}-u_{2}
$$

is the solution of the following Cauchy problem:

$$
\begin{cases}\partial_{t} \omega+\kappa \partial_{x}\left(u_{1}^{2}-u_{2}^{2}\right)-\beta^{2} \partial_{x}^{6} \omega+\alpha \partial_{x}^{4} \omega+\delta^{2} \partial_{x}^{4}\left(u_{1}^{3}-u_{2}^{3}\right)=0, & t>0, x \in \mathbb{R}, \\ \omega(0, x)=u_{1,0}(x)-u_{2,0}(x), & x \in \mathbb{R} .\end{cases}
$$

Since

$$
\begin{aligned}
2 \int_{\mathbb{R}} \omega \partial_{t} \omega d x & =\frac{d}{d t}\|\omega(t, \cdot)\|_{L^{2}(\mathbb{R})}^{2}, \\
2 \kappa \int_{\mathbb{R}} \partial_{x}\left(u_{1}^{2}-u_{2}^{2}\right) \omega d x & =-2 \kappa \int_{\mathbb{R}}\left(u_{1}^{2}-u_{2}^{2}\right) \partial_{x} \omega d x, \\
-2 \beta^{2} \int_{\mathbb{R}} \omega \partial_{x}^{6} \omega d x & =2 \beta^{2}\left\|\partial_{x}^{3} \omega(t, \cdot)\right\|_{L^{2}(\mathbb{R})^{\prime}}^{2} \\
2 \alpha \int_{\mathbb{R}} \omega \partial_{x}^{4} \omega d x & =-2 \alpha \int_{\mathbb{R}} \partial_{x} \omega \partial_{x}^{3} \omega d x, \\
2 \delta^{2} \int_{\mathbb{R}} \omega \partial_{x}^{4}\left(u_{1}^{3}-u_{2}^{3}\right) d x & =-2 \delta^{2} \int_{\mathbb{R}} \partial_{x}^{3} \omega \partial_{x}\left(u_{1}^{3}-u_{2}^{3}\right) d x,
\end{aligned}
$$


multiplying (66) by $2 \omega$, an integration on $\mathbb{R}$ gives

$$
\begin{aligned}
& \frac{d}{d t}\|\omega(t, \cdot)\|_{L^{2}(\mathbb{R})}^{2}+2 \beta^{2}\left\|\partial_{x}^{3} \omega(t, \cdot)\right\|_{L^{2}(\mathbb{R})}^{2} \\
& \quad=2 \kappa \int_{\mathbb{R}}\left(u_{1}^{2}-u_{2}^{2}\right) \partial_{x} \omega d x-2 \alpha \int_{\mathbb{R}} \partial_{x} \omega \partial_{x}^{3} \omega d x+2 \delta^{2} \int_{\mathbb{R}} \partial_{x}^{3} \omega \partial_{x}\left(u_{1}^{3}-u_{2}^{3}\right) d x .
\end{aligned}
$$

Observe that thanks to (65),

$$
u_{1}^{2}-u_{2}^{2}=\left(u_{1}+u_{2}\right) \omega, \quad u_{1}^{3}-u_{2}^{3}=\left(u_{1}^{2}+u_{2}+u_{1} u_{2}\right) \omega .
$$

Consequently, by (67),

$$
\begin{aligned}
\frac{d}{d t}\|\omega(t, \cdot)\|_{L^{2}(\mathbb{R})}^{2}+2 \beta^{2}\left\|\partial_{x}^{3} \omega(t, \cdot)\right\|_{L^{2}(\mathbb{R})}^{2} \\
=2 \kappa \int_{\mathbb{R}}\left(u_{1}+u_{2}\right) \omega \partial_{x} \omega d x-2 \alpha \int_{\mathbb{R}} \partial_{x} \omega \partial_{x}^{3} \omega d x \\
\quad+2 \delta^{2} \int_{\mathbb{R}} \partial_{x}^{3} \omega \partial_{x}\left(\left(u_{1}^{2}+u_{2}+u_{1} u_{2}\right) \omega\right) d x \\
=-\kappa \int_{\mathbb{R}}\left(\partial_{x} u_{1}+\partial_{x} u_{2}\right) \omega^{2} d x-2 \alpha \int_{\mathbb{R}} \partial_{x} \omega \partial_{x}^{3} \omega d x \\
\quad+2 \delta^{2} \int_{\mathbb{R}}\left(2 u_{1} \partial_{x} u_{1}+2 u_{2} \partial_{x} u_{2}+u_{2} \partial_{x} u_{1}+u_{1} \partial_{x} u_{2}\right) \omega \partial_{x}^{3} \omega d x \\
\quad+2 \delta^{2} \int_{\mathbb{R}}\left(u_{1}^{2}+u_{2}+u_{1} u_{2}\right) \partial_{x} \omega \partial_{x}^{3} \omega d x .
\end{aligned}
$$

Observe that since $u_{1}, u_{2} \in H^{3}(\mathbb{R})$, for every $0 \leq t \leq T$, we have that

$$
\begin{aligned}
& \left\|u_{1}\right\|_{L^{\infty}((0, T) \times \mathbb{R})},\left\|\partial_{x} u_{1}\right\|_{L^{\infty}((0, T) \times \mathbb{R})} \leq C(T), \\
& \left\|u_{2}\right\|_{L^{\infty}((0, T) \times \mathbb{R})},\left\|\partial_{x} u_{2}\right\|_{L^{\infty}((0, T) \times \mathbb{R})} \leq C(T) .
\end{aligned}
$$

Consequently, thanks to (70), we obtain that

$$
\begin{aligned}
&\left|\partial_{x} u_{1}+\partial_{x} u_{2}\right| \leq C(T), \\
&\left|2 u_{1} \partial_{x} u_{1}+2 u_{2} \partial_{x} u_{2}+u_{2} \partial_{x} u_{1}+u_{1} \partial_{x} u_{2}\right| \leq C(T), \\
&\left|u_{1}^{2}+u_{2}+u_{1} u_{2}\right| \leq C(T) .
\end{aligned}
$$

Due to (71) and the Young inequality,

$$
\begin{aligned}
& |\kappa| \int_{\mathbb{R}}\left|\partial_{x} u_{1}+\partial_{x} u_{2}\right| \omega^{2} d x \leq C(T)\|\omega(t, \cdot)\|_{L^{2}(\mathbb{R})}^{2}, \\
& 2|\alpha| \int_{\mathbb{R}}\left|\partial_{x} \omega \| \partial_{x}^{3} \omega\right| d x=\int_{\mathbb{R}}\left|\frac{2 \alpha \partial_{x} \omega}{\beta}\right|\left|\beta \partial_{x}^{3} \omega\right| d x \\
& \leq \frac{2 \alpha^{2}}{\beta^{2}}\left\|\partial_{x} \omega(t, \cdot)\right\|_{L^{2}(\mathbb{R})}^{2}+\frac{\beta^{2}}{2}\left\|\partial_{x}^{3} \omega(t, \cdot)\right\|_{L^{2}(\mathbb{R})^{\prime}}^{2} \\
& 2 \delta^{2} \int_{\mathbb{R}}\left|2 u_{1} \partial_{x} u_{1}+2 u_{2} \partial_{x} u_{2}+u_{2} \partial_{x} u_{1}+u_{1} \partial_{x} u_{2}\right||\omega|\left|\partial_{x}^{3} \omega\right| d x \\
& \quad \leq C(T) \int_{\mathbb{R}}|\omega|\left|\partial_{x}^{3} \omega\right| d x=\int_{\mathbb{R}}\left|\frac{C(T) \omega}{\beta}\right||| \beta \partial_{x}^{3} \mid d x \\
& \leq C(T)\|\omega(t, \cdot)\|_{L^{2}(\mathbb{R})}^{2}+\frac{\beta^{2}}{2}\left\|\partial_{x}^{3} \omega(t, \cdot)\right\|_{L^{2}(\mathbb{R})^{\prime}}^{2} \\
& 2 \delta^{2} \int_{\mathbb{R}}\left|u_{1}^{2}+u_{2}+u_{1} u_{2}\right|\left|\partial_{x} \omega \| \partial_{x}^{3} \omega\right| d x
\end{aligned}
$$




$$
\begin{aligned}
& \leq C(T) \int_{\mathbb{R}}\left|\partial_{x} \omega \| \partial_{x}^{3} \omega\right| d x=\int_{\mathbb{R}}\left|\frac{C(T) \partial_{x} \omega}{\beta}\right|\left|\beta \partial_{x}^{3} u\right| d x \\
& \leq C(T)\left\|\partial_{x} \omega(t, \cdot)\right\|_{L^{2}(\mathbb{R})}^{2}+\frac{\beta^{2}}{2}\left\|\partial_{x}^{3} \omega(t, \cdot)\right\|_{L^{2}(\mathbb{R})}^{2} .
\end{aligned}
$$

It follows from (69) that

$$
\begin{aligned}
& \frac{d}{d t}\|\omega(t, \cdot)\|_{L^{2}(\mathbb{R})}^{2}+\frac{\beta^{2}}{2}\left\|\partial_{x}^{3} \omega(t, \cdot)\right\|_{L^{2}(\mathbb{R})}^{2} \\
& \leq C(T)\|\omega(t, \cdot)\|_{L^{2}(\mathbb{R})}^{2}+C(T)\left\|\partial_{x} \omega(t, \cdot)\right\|_{L^{2}(\mathbb{R})}^{2} .
\end{aligned}
$$

Observe that

$$
C(T)\left\|\partial_{x} \omega(t, \cdot)\right\|_{L^{2}(\mathbb{R})}^{2}=C(T) \int_{\mathbb{R}} \partial_{x} \omega \partial_{x} \omega d x=-C(T) \int_{\mathbb{R}} \omega \partial_{x}^{2} \omega d x .
$$

Therefore, by the Young inequality,

$$
\begin{aligned}
C(T)\left\|\partial_{x} \omega(t, \cdot)\right\|_{L^{2}(\mathbb{R})}^{2} & \leq C(T) \int_{\mathbb{R}}\left|\frac{\omega}{\sqrt{D_{5}}}\right|\left|\sqrt{D_{5}} \partial_{x}^{2} \omega\right| d x \\
& \leq \frac{C(T)}{2 D_{5}}\|\omega(t, \cdot)\|_{L^{2}(\mathbb{R})}^{2}+\frac{C(T) D_{5}}{2}\left\|\partial_{x}^{2} \omega(t, \cdot)\right\|_{L^{2}(\mathbb{R})}^{2}
\end{aligned}
$$

where $D_{5}$ is a positive constant, which will specified later. Observe again that

$$
\frac{C(T) D_{5}}{2}\left\|\partial_{x}^{2} \omega(t, \cdot)\right\|_{L^{2}(\mathbb{R})}^{2}=\frac{C(T) D_{5}}{2} \int_{\mathbb{R}} \partial_{x}^{2} \omega \partial_{x}^{2} \omega d x=-\frac{C(T) D_{5}}{2} \int_{\mathbb{R}} \partial_{x} \omega \partial_{x}^{3} \omega d x .
$$

Consequently, by the Young inequality,

$$
\begin{aligned}
& \frac{C(T) D_{5}}{2}\left\|\partial_{x}^{2} \omega(t, \cdot)\right\|_{L^{2}(\mathbb{R})}^{2} \leq \frac{C(T) D_{5}}{2} \int_{\mathbb{R}}\left|\frac{\partial_{x} \omega}{\sqrt{D_{6}}}\right|\left|\sqrt{D_{6}} \partial_{x}^{3} \omega\right| d x \\
& \leq \frac{C(T) D_{5}}{4 D_{6}}\left\|\partial_{x} \omega(t, \cdot)\right\|_{L^{2}(\mathbb{R})}^{2}+\frac{C(T) D_{5} D_{6}}{4}\left\|\partial_{x}^{3} \omega(t, \cdot)\right\|_{L^{2}(\mathbb{R})}^{2}
\end{aligned}
$$

where $D_{6}$ is a positive constant, which will specified later. It follows from (73) and (74) that

$$
C(T)\left(1-\frac{D_{5}}{4 D_{6}}\right)\left\|\partial_{x} \omega(t, \cdot)\right\|_{L^{2}(\mathbb{R})}^{2} \leq \frac{C(T)}{2 D_{5}}\|\omega(t, \cdot)\|_{L^{2}(\mathbb{R})}^{2}+\frac{C(T) D_{5} D_{6}}{4}\left\|\partial_{x}^{3} \omega(t, \cdot)\right\|_{L^{2}(\mathbb{R})}^{2} .
$$

Taking

$$
D_{5}=2 D_{6}
$$

We have

$$
\frac{C(T)}{2}\left\|\partial_{x} \omega(t, \cdot)\right\|_{L^{2}(\mathbb{R})}^{2} \leq \frac{C(T)}{4 D_{6}}\|\omega(t, \cdot)\|_{L^{2}(\mathbb{R})}^{2}+\frac{C(T) D_{6}^{2}}{2}\left\|\partial_{x}^{3} \omega(t, \cdot)\right\|_{L^{2}(\mathbb{R})}^{2} .
$$

Therefore,

$$
C(T)\left\|\partial_{x} \omega(t, \cdot)\right\|_{L^{2}(\mathbb{R})}^{2} \leq \frac{C(T)}{D_{6}}\|\omega(t, \cdot)\|_{L^{2}(\mathbb{R})}^{2}+C(T) D_{6}^{2}\left\|\partial_{x}^{3} \omega(t, \cdot)\right\|_{L^{2}(\mathbb{R})}^{2} .
$$

It follows from (72) and (75) that

$$
\frac{d}{d t}\|\omega(t, \cdot)\|_{L^{2}(\mathbb{R})}^{2}+\left(\frac{\beta^{2}}{2}-C(T) D_{6}^{2}\right)\left\|\partial_{x}^{3} \omega(t, \cdot)\right\|_{L^{2}(\mathbb{R})}^{2} \leq C(T)\left(1+\frac{1}{D_{6}}\right)\|\omega(t, \cdot)\|_{L^{2}(\mathbb{R})}^{2} .
$$


Choosing

$$
D_{6}=\frac{|\beta|}{\sqrt{3 C(T)}}
$$

we have that

$$
\frac{d}{d t}\|\omega(t, \cdot)\|_{L^{2}(\mathbb{R})}^{2}+\frac{\beta^{2}}{6}\left\|\partial_{x}^{3} \omega(t, \cdot)\right\|_{L^{2}(\mathbb{R})}^{2} \leq C(T)\|\omega(t, \cdot)\|_{L^{2}(\mathbb{R})}^{2}
$$

The Gronwall Lemma and (66) gives

$$
\|\omega(t, \cdot)\|_{L^{2}(\mathbb{R})}^{2}+\frac{\beta^{2} e^{C(T) t}}{6} \int_{0}^{t} e^{-C(T) s}\left\|\partial_{x}^{3} \omega(s, \cdot)\right\|_{L^{2}(\mathbb{R})}^{2} d s \leq e^{C(T) t}\left\|\omega_{0}\right\|_{L^{2}(\mathbb{R})} .
$$

(10) follows from (65) and (76).

\section{Conclusions}

We considered the high order convective Cahn-Hilliard type equations that describe the faceting of a growing surface, or the dynamics of phase transitions in ternary oil-water-surfactant systems. We proved the well-posedness of the We considered the Cauchy problem and proved its well-posedness when the initial condition has zero mean and belongs to $H^{3}$.

Author Contributions: Conceptualization, G.M.C. and L.d.R.; Methodology, G.M.C. and L.d.R.; Software, G.M.C. and L.d.R.; Validation, G.M.C. and L.d.R.; Formal analysis, G.M.C. and L.d.R.; Investigation, G.M.C. and L.d.R.; Resources, G.M.C. and L.d.R.; Data curation, G.M.C. and L.d.R.; Writing-original draft preparation, G.M.C. and L.d.R.; Writing-review and editing, G.M.C. and L.d.R.; Visualization, G.M.C. and L.d.R.; Supervision, G.M.C. and L.d.R.; Project administration, G.M.C. and L.d.R.; Funding acquisition, G.M.C. and L.d.R. All authors have read and agreed to the published version of the manuscript.

Funding: This research received no external funding.

Acknowledgments: The authors are members of the Gruppo Nazionale per l'Analisi Matematica, la Probabilità e le loro Applicazioni (GNAMPA) of the Istituto Nazionale di Alta Matematica (INdAM).

Conflicts of Interest: The authors declare no conflict of interest.

\section{References}

1. Coclite, G.M.; di Ruvo, L. Well-posedness of bounded solutions of the non-homogeneous initial-boundary value problem for the Ostrovsky-Hunter equation. J. Hyperbolic Differ. Equ. 2015, 12, 221-248. [CrossRef]

2. Coclite, G.M.; di Ruvo, L. Oleinik type estimates for the Ostrovsky-Hunter equation. J. Math. Anal. Appl. 2015, 423, 162-190. [CrossRef]

3. Coclite, G.M.; di Ruvo, L. Convergence of the Ostrovsky equation to the Ostrovsky-Hunter one. J. Differ. Equ. 2014, 256, 3245-3277. [CrossRef]

4. Coclite, G.M.; di Ruvo, L. Well-posedness results for the short pulse equation. Z. Angew. Math. Phys. 2015, 66, 1529-1557. [CrossRef]

5. Coclite, G.M.; di Ruvo, L. Wellposedness of bounded solutions of the non-homogeneous initial boundary for the short pulse equation. Boll. Unione Mat. Ital. 2015, 8, 31-44. [CrossRef]

6. Coclite, G.M.; di Ruvo, L. Convergence of the regularized short pulse equation to the short pulse one. Math. Nachr. 2018, 291, 774-792. [CrossRef]

7. Coclite, G.M.; di Ruvo, L. Well-posedness and dispersive/diffusive limit of a generalized Ostrovsky-Hunter equation. Milan J. Math. 2018, 86, 31-51. [CrossRef]

8. Coclite, G.M.; di Ruvo, L. A note on the non-homogeneous initial boundary problem for an Ostrovsky-Hunter type equation. Discrete Contin. Dyn. Syst. Ser. S 2020. [CrossRef]

9. Coclite, G.M.; di Ruvo, L. A non-local regularization of the short pulse equation. Minimax Theory Appl. 2020, in press. [CrossRef]

10. Coclite, G.M.; di Ruvo, L. A non-local elliptic-hyperbolic system related to the short pulse equation. Nonlinear Anal. 2020, 190, 111606. [CrossRef]

11. Coclite, G.M.; di Ruvo, L. Well-Posedness Results for the Continuum Spectrum Pulse Equation. Mathematics 2019, 7, 1006. [CrossRef] 
12. Coclite, G.M.; di Ruvo, L. On the solutions for an Ostrovsky type equation. Nonlinear Anal. Real World Appl. 2020, 55, 103141. [CrossRef]

13. Savina, T.V.; Golovin, A.A.; Davis, S.H.; Nepomnyashchy, A.A.; Voorhees, P.W. Faceting of a growing crystal surface by surface diffusion. Phys. Rev. E 2003, 67, 021606. [CrossRef] [PubMed]

14. Barakat, F.; Martens, K.; Pierre-Louis, O. Nonlinear Wavelength Selection in Surface Faceting under Electromigration. Phys. Rev. Lett. 2012, 109, 056101. [CrossRef] [PubMed]

15. Berry, J.; Elder, K.R.; Grant, M. Simulation of an atomistic dynamic field theory for monatomic liquids: Freezing and glass formation. Phys. Rev. E 2008, 77, 061506. [CrossRef]

16. Berry, J.; Grant, M.; Elder, K.R. Diffusive atomistic dynamics of edge dislocations in two dimensions. Phys. Rev. E 2006, 73, 031609. [CrossRef]

17. Dlotko, T.; Kania, M.B.; Sun, C. Analysis of the viscous Cahn-Hilliard equation in $\mathbb{R}^{N}$. J. Differ. Equ. 2012, 252, 2771-2791. [CrossRef]

18. Elder, K.R.; Grant, M. Modeling elastic and plastic deformations in nonequilibrium processing using phase field crystals. Phys. Rev. E 2004, 70, 051605. [CrossRef]

19. Elder, K.R.; Katakowski, M.; Haataja, M.; Grant, M. Modeling Elasticity in Crystal Growth. Phys. Rev. Lett. 2002, 88, 245701. [CrossRef]

20. Goldstein, G.R.; Miranville, A.; Schimperna, G. A Cahn-Hilliard model in a domain with non-permeable walls. Phys. D Nonlinear Phenom. 2011, 240, 754-766. [CrossRef]

21. Golovin, A.A.; Nepomnyashchy, A.A.; Davis, S.H.; Zaks, M.A. Convective Cahn-Hilliard Models: From Coarsening to Roughening. Phys. Rev. Lett. 2001, 86, 1550-1553. [CrossRef] [PubMed]

22. Watson, S.J.; Otto, F.; Rubinstein, B.Y.; Davis, S.H. Coarsening dynamics of the convective Cahn-Hilliard equation. Phys. D 2003, 178, 127-148. [CrossRef]

23. Korzec, M.D.; Rybka, P. On a higher order convective Cahn-Hilliard-type equation. SIAM J. Appl. Math. 2012, 72, 1343-1360. [CrossRef]

24. Korzec, M.D.; Nayar, P.; Rybka, P. Global weak solutions to a sixth order Cahn-Hilliard type equation. SIAM J. Math. Anal. 2012, 44, 3369-3387. [CrossRef]

25. Korzec, M.D.; Evans, P.L.; Münch, A.; Wagner, B. Stationary solutions of driven fourth- and sixth-order Cahn-Hilliard-type equations. SIAM J. Appl. Math. 2008, 69, 348-374. [CrossRef]

26. Zhao, X.; Duan, N. Optimal control of the sixth-order convective Cahn-Hilliard equation. Bound. Value Probl. 2014, 2014, 206. [CrossRef]

27. Korzec, M.D.; Nayar, P.; Rybka, P. Global attractors of sixth order PDEs describing the faceting of growing surfaces. J. Dynam. Differ. Equ. 2016, 28, 49-67. [CrossRef]

28. Gompper, G.; Goos, J. Fluctuating interfaces in microemulsion and sponge phases. Phys. Rev. E 1994, 50, 1325-1335. [CrossRef]

29. Gompper, G.; Kraus, M. Ginzburg-Landau theory of ternary amphiphilic systems. I. Gaussian interface fluctuations. Phys. Rev. E 1993, 47, 4289-4300. [CrossRef]

30. Gompper, G.; Kraus, M. Ginzburg-Landau theory of ternary amphiphilic systems. II. Monte Carlo simulations. Phys. Rev. E 1993, 47, 4301-4312. [CrossRef]

31. Pawł ow, I.; Zajączkowski, W.M. A sixth order Cahn-Hilliard type equation arising in oil-water-surfactant mixtures. Commun. Pure Appl. Anal. 2011, 10, 1823-1847. [CrossRef]

32. Liu, A.; Liu, C. Weak solutions for a sixth order Cahn-Hilliard type equation with degenerate mobility. Abstr. Appl. Anal. 2014, 2014, 407265. [CrossRef]

33. Topper, J.; Kawahara, T. Approximate equations for long nonlinear waves on a viscous fluid. J. Phys. Soc. Jpn. 1978, 44, 663-666. [CrossRef]

34. Cohen, B.; Krommes, J.; Tang, W.; Rosenbluth, M. Non-linear saturation of the dissipative trapped-ion mode by mode coupling. Nucl. Fusion 1976, 16, 971-992. [CrossRef]

35. Kuramoto, Y. Diffusion-Induced Chaos in Reaction Systems. Prog. Theor. Phys. Suppl. 1978, 64, $346-367$. [CrossRef]

36. Kuramoto, Y.; Tsuzuki, T. On the Formation of Dissipative Structures in Reaction-Diffusion Systems: Reductive Perturbation Approach. Prog. Theor. Phys. 1975, 54, 687-699. [CrossRef]

37. Kuramoto, Y.; Tsuzuki, T. Persistent Propagation of Concentration Waves in Dissipative Media Far from Thermal Equilibrium. Prog. Theor. Phys. 1976, 55, 356-369. [CrossRef] 
38. Sivashinsky, G.I. Nonlinear analysis of hydrodynamic instability in laminar flames. I. Derivation of basic equations. Acta Astronaut. 1977, 4, 1177-1206. [CrossRef]

39. Chen, L.H.; Chang, H.C. Nonlinear waves on liquid film surfaces-II. Bifurcation analyses of the long-wave equation. Chem. Eng. Sci. 1986, 41, 2477-2486. [CrossRef]

40. Hooper, A.P.; Grimshaw, R. Nonlinear instability at the interface between two viscous fluids. Phys. Fluids 1985, 28, 37-45. [CrossRef]

41. LaQuey, R.E.; Mahajan, S.M.; Rutherford, P.H.; Tang, W.M. Nonlinear Saturation of the Trapped-Ion Mode. Phys. Rev. Lett. 1975, 34, 391-394. [CrossRef]

42. Benney, D.J. Long waves on liquid films. J. Math. Phys. 1966, 45, 150-155. [CrossRef]

43. Lin, S.P. Finite amplitude side-band stability of a viscous film. J. Fluid Mech. 1974, 63, 417-429. [CrossRef]

44. Li, C.; Chen, G.; Zhao, S. Exact travelling wave solutions to the generalized Kuramoto-Sivashinsky equation. Lat. Am. Appl. Res. 2004, 34, 65-68.

45. Foias, C.; Nicolaenko, B.; Sell, G.R.; Temam, R. Inertial manifolds for the Kuramoto-Sivashinsky equation and an estimate of their lowest dimension. J. Math. Pures Appl. 1988, 67, 197-226.

46. Khalique, C. Exact Solutions of the Generalized Kuramoto-Sivashinsky Equation. Casp. J. Math. Sci. (CJMS) 2012, 1, 109-116.

47. Kudryashov, N.A. Exact solutions of the generalized Kuramoto-Sivashinsky equation. Phys. Lett. A 1990, 147, 287-291. [CrossRef]

48. Nicolaenko, B.; Scheurer, B. Remarks on the Kuramoto-Sivashinsky equation. Phys. D 1984, 12, $391-395$. [CrossRef]

49. Nicolaenko, B.; Scheurer, B.; Temam, R. Some global dynamical properties of the Kuramoto-Sivashinsky equations: Nonlinear stability and attractors. Phys. D 1985, 16, 155-183. [CrossRef]

50. Xie, Y. Solving the generalized Benney equation by a combination method. Int. J. Nonlinear Sci. 2013, 15, 350-354.

51. Armaou, A.; Christofides, P.D. Feedback control of the Kuramoto-Sivashinsky equation. Phys. D 2000, 137, 49-61. [CrossRef]

52. Cerpa, E. Null controllability and stabilization of the linear Kuramoto-Sivashinsky equation. Commun. Pure Appl. Anal. 2010, 9, 91-102. [CrossRef]

53. Giacomelli, L.; Otto, F. New bounds for the Kuramoto-Sivashinsky equation. Comm. Pure Appl. Math. 2005, 58, 297-318. [CrossRef]

54. Christofides, P.D.; Armaou, A. Global stabilization of the Kuramoto-Sivashinsky equation via distributed output feedback control. Syst. Control Lett. 2000, 39, 283-294. [CrossRef]

55. Hu, C.; Temam, R. Robust control of the Kuramoto-Sivashinsky equation. Dyn. Contin. Discret. Impuls. Syst. Ser. B Appl. Algorithms 2001, 8, 315-338.

56. Liu, W.J.; Krstić, M. Stability enhancement by boundary control in the Kuramoto-Sivashinsky equation. Nonlinear Anal. 2001, 43, 485-507. [CrossRef]

57. Sajjadian, M. The shock profile wave propagation of Kuramoto-Sivashinsky equation and solitonic solutions of generalized Kuramoto-Sivashinsky equation. Acta Univ. Apulensis Math. Inform. 2014, 38, 163-176.

58. Biagioni, H.A.; Bona, J.L.; Iório, R.J., Jr.; Scialom, M. On the Korteweg-de Vries-Kuramoto-Sivashinsky equation. Adv. Differ. Equ. 1996, 1, 1-20.

59. Coclite, G.M.; di Ruvo, L. On Classical Solutions for A Kuramoto-Sinelshchikov-Velarde-Type Equation. Algorithms 2020, 13, 77. [CrossRef]

60. Tadmor, E. The well-posedness of the Kuramoto-Sivashinsky equation. SIAM J. Math. Anal. 1986, 17, 884-893. [CrossRef]

61. Coclite, G.M.; di Ruvo, L. Dispersive and diffusive limits for Ostrovsky-Hunter type equations. NoDEA Nonlinear Differ. Equ. Appl. 2015, 22, 1733-1763. [CrossRef]

62. LeFloch, P.G.; Natalini, R. Conservation laws with vanishing nonlinear diffusion and dispersion. Nonlinear Anal. 1999, 36, 213-230. [CrossRef]

63. Schonbek, M.E. Convergence of solutions to nonlinear dispersive equations. Comm. Partial Differ. Equ. 1982, 7, 959-1000. [CrossRef]

64. Coclite, G.M.; di Ruvo, L. Convergence of the Kuramoto-Sinelshchikov equation to the Burgers one. Acta Appl. Math. 2016, 145, 89-113. [CrossRef] 
65. Wang, Z.; Liu, C. Some properties of solutions for the sixth-order Cahn-Hilliard-type equation. Abstr. Appl. Anal. 2012, 2012, 414590. [CrossRef]

66. Coclite, G.M.; di Ruvo, L. Existence results for the Kudryashov-Sinelshchikov-Olver equation. Proc. R. Soc. Edinb. Sect. A Math. 2020, 1-26. [CrossRef]

67. Taylor, M.E. Partial Differential Equations I. Basic Theory, 2nd ed.; Springer: New York, NY, USA, 2011; Volume 115, pp. xxii+654. [CrossRef]

(C) 2020 by the authors. Licensee MDPI, Basel, Switzerland. This article is an open access article distributed under the terms and conditions of the Creative Commons Attribution (CC BY) license (http:/ / creativecommons.org/licenses/by/4.0/). 